\title{
REPRESENTATIONS OF GENERIC ALGEBRAS AND FINITE GROUPS OF LIE TYPE
}

BY

\author{
R. B. HOWLETT AND G. I. LEHRER
}

\begin{abstract}
The complex representation theory of a finite Lie group $G$ is related to that of certain "generic algebras". As a consequence, formulae are derived ("the Comparison Theorem"), relating multiplicities in $G$ to multiplicities in the Weyl group $W$ of $G$. Applications include an explicit description of the dual (see below) of an arbitrary irreducible complex representation of $G$
\end{abstract}

Introduction. Let $G$ be the group of $\mathbf{F}_{q}$-rational points of a (connected) reductive $\mathbf{F}_{q}$-group $G$, where $\mathbf{F}_{q}$ is the Galois field of $q$ elements. The purpose of this paper is to study the complex representation theory of $G$ using the "generic algebra" techniques hinted at in our earlier paper [9], to which the present work may be considered a sequel. For any unexplained notation, the reader is referred to [9]. Our main theorem (5.9) compares inner products of certain characters of $G$ with corresponding inner products within its Weyl group $W$. As an application of this "comparison theorem" we determine precisely what the dual (cf. Curtis [3]) of an arbitrary irreducible character of $G$ is.

In order to describe our results explicitly, we mention the principal facts concerning the structure of $G$ which we shall require. Firstly, $G$ has a split ( $B, N)$-pair in characteristic $p$ (where $q=p^{e}$ ). Secondly, each standard parabolic subgroup $P_{J}$ of $G$ (where $J$ is a subset of the simple roots associated with the ( $B, N)$-structure) has a standard Levi decomposition $P_{J}=M_{J} U_{J}$, where $U_{J}$ is the "unipotent radical" of $P_{J}$, and $M_{J}$, the standard Levi component of $P_{J}$, is again the group of rational points of a reductive $\mathbf{F}_{q}$-group. For more details, the reader is referred to $[\mathbf{9}, \S 1]$.

The passage between the representation theory of $G$ and of its Weyl group $W$ is accomplished by means of certain "generic algebras", which are associative algebras over a complex polynomial ring and which "specialize" to various complex algebras which have significance for the representation theory of $G$ and $W$. More precisely, the "Harish-Chandra principle" (see $[9, \S 1]$ or $\S 3$ below) organizes the representations of $G$ into "series" which consist of the irreducible constituents of a fixed induced cuspidal representation $\operatorname{Ind}\left(P_{L} \rightarrow G ; \tilde{D}\right)$ (where $P_{L}$ is a parabolic subgroup of $G$ and $D$ is a cuspidal representation of $M_{L}$ ). In [9], the constituents of a single series were studied by elucidating the structure of the endomorphism algebra of $\operatorname{Ind}\left(P_{L} \rightarrow G ; \tilde{D}\right)$. In $\S 1$ of the present work we make explicit the connection between the decomposition of induced representations and the representation theory of the

Received by the editors December 20, 1982.

1980 Mathematics Subject Classification. Primary 20G05, 20G40; Secondary 16A64, 16A65.

1983 American Mathematical Society $0002-9947 / 83 \$ 1.00+\$ .25$ per page 
associated endomorphism algebras by introducing two pairs of adjoint functors and studying the way they interact. This transfers the study of any particular series to the study of a certain endomorphism algebra.

The principal result of [9] (see $\$ 3$ below) was that the endomorphism algebras concerned have an explicit presentation in terms of the ramification group $W(D)$ (see $\$ 2$ below) of $D$ and certain powers $p_{a}$ of the characteristic. Replacing these $p_{a}$ by indeterminates $u_{a}$, one constructs "generic algebras" over the ring $\mathbf{C}\left[\left\{u_{a}\right\}\right]$; $\$ 4$ of the present work is devoted to the study of these generic algebras and of the connection between their representation theory and that of their various specializations, among which are twisted group algebras of the ramification groups $W(D)$. This provides (Theorem (4.8)) explicit descriptions of each of the series of representations of $G$, in terms of data relating to the cuspidal representation and its ramification group.

In $\$ 5$ a refinement (5.6) of this description is proved, in which $G$ is replaced by the Levi component $M_{J}$ of the parabolic subgroup $P_{J}$. In addition, certain geometric results concerning the ramification groups and their representation theory are given, which in $\S 6$ and $\S 7$ are transported into results concerning $G$.

In general terms, the Comparison Theorem (5.9) states that if $\phi$ and $\psi$ are characters of appropriate projective representations of the ramification group $W(\delta)$ and its subgroup $W_{J}^{w} \cap W(\delta)$ (where $w L \subset J \subset \Pi$ and $\delta$ is an irreducible cuspidal character of $M_{L}$ ), and $\zeta_{\phi}, \eta_{u \psi \psi}$ are the corresponding characters in the $\delta$-series of $G$ and $w \delta$-series of $M_{J}$ respectively, then the multiplicities of $\zeta_{\phi} \operatorname{in} \operatorname{Ind}\left(P_{J} \rightarrow G ; \tilde{\eta}_{\text {x }^{\prime} \psi}\right)$ and of $\phi$ in $\operatorname{Ind}\left(W_{J}^{w} \cap W(\delta) \rightarrow W(\delta) ; \psi\right)$ are equal. The proof is accomplished by showing that both multiplicities are equal to a certain multiplicity which arises in the generic algebra context. En route, one encounters a certain subalgebra $A_{J, w}$ of the generic algebra which is related to the representation theory of $M_{J}$, but which needs to be "conjugated" so that its specializations may be explicitly identified. Hence the necessity for $\S 3$, where the results of [9] are recalled and slightly generalized.

The Duality Theorem (7.5) shows that with the above notation, the dual $\zeta_{\phi}^{*}$ of $\zeta_{\phi}$ is equal to $(-1)^{\mid I_{1}} \zeta_{\alpha \phi}$ where $\alpha$ is a certain one-dimensional character of $W(\delta)$ which is described geometrically (see (5.2)) and which reduces to the sign character of $W$ in the case of principal series. Thus our theorem generalizes the results of Curtis [3] who studied the case of principal series $\left(1_{B}^{G}\right)$ and McGovern [12], who treated the case of generalized principal series, where $L$ is empty but $\delta \neq 1$. These two authors also proved results corresponding to the comparison theorem in their respective situations, as did Lusztig [11] for the case where $\delta$ is a unipotent cuspidal representation. The major results of the present work were announced in [7].

1. Endormorphism algebras and induced modules. In this section we develop certain results connecting the submodules of a module with representations of its endomorphism algebra. These results are applied to study endomorphism algebras of induced modules, in particular to prove a "strong Frobenius reciprocity theorem" (1.13) which asserts that two functors commute. Most results in this section are rather familiar, and we shall therefore give only sketchy proofs.

Let $V$ be a (unital) module for a complex semisimple algebra $A$. We assume that $\operatorname{dim}_{\mathbf{C}} V$ is finite, and (w.l.o.g., since $A$ may be replaced by its enveloping algebra in 
End $_{\mathrm{C}} V$ ) that $A$ acts faithfully on $V$. Let $E=\operatorname{End}_{A} V$, and write elements of $E$ as left operators on $V$. Denote by $Q$ and $E$ the categories of left $A$ - and $E$-modules respectively. We define contravariant functors $\mathfrak{F}: \mathscr{Q} \rightarrow \mathcal{E}$ and $\mathfrak{F}^{\prime}: \mathscr{E} \rightarrow \mathscr{Q}$ as follows:

(1.1) Definition. (i) For $M \in \mathbb{Q}$, define $\mathfrak{F}(M)=\operatorname{Hom}_{A}(M, V)$. The $E$-action on $\mathfrak{F}(M)$ is defined by

$$
(e \phi)(m)=e(\phi(m)) \quad(e \in E, m \in M, \phi \in \mathfrak{F}(M)) .
$$

(ii) For $N \in \mathbb{E}$, define $\widetilde{F}^{\prime}(N)=\operatorname{Hom}_{E}(N, V)$. The $A$-action is defined by

$$
(a \psi)(n)=a(\psi(n)) \quad\left(a \in A, n \in N, \psi \in \mathcal{F}^{\prime}(N)\right) .
$$

We leave it to the reader to check that these definitions yield contravariant functors as claimed.

(1.2) LEMMA. We have

(i) $\mathfrak{T}(V) \cong E$,

(ii) $\widetilde{r}^{\prime}(V) \cong A$,

(iii) $\mathfrak{\text { T }}(A) \cong V$,

(iv) $\pi^{\prime}(E) \cong V$,

where in (i) and (iii) the isomorphisms are in (E), while in (ii) and (iv) the isomorphisms are in ( $(1$ '.

Proof. Part (i) is immediate from the definition of $E$ and (ii) follows from Wedderburn's Theorem (see [6, $\$ 26.8$, example 1]). The isomorphism in (iii) is given as follows: for each $v \in V$ define $\theta_{v^{*}} \in \mathfrak{T}(A)$ by $\phi_{v}(a)=a v$. It is easily checked that $v \mapsto \theta_{v}$ defines on $E$-isomorphism: $V \rightarrow \mathfrak{F}(A)$. The proof of (iv) is similar.

(1.3) Lemma. (i) If $M_{1}, M_{2} \in \mathbb{Q}$, then $\mathfrak{F}\left(M_{1} \oplus M_{2}\right) \cong \mathfrak{F}\left(M_{1}\right) \oplus \mathfrak{F}\left(M_{2}\right)$ (i.e. ₹r is additive).

(ii) If $M \in Q$ is irreducible then so is $\mathfrak{F}(M)$.

Proof. (i) is trivial. For (ii), suppose $M \in Q$ is irreducible. Since $V$ is faithful, it has a component isomorphic to $M$, whence $\mathfrak{F}(M)=\operatorname{Hom}_{A}(M, V) \neq 0$. Take any nonzero element $f \in \mathcal{T}(M)$. Since $M$ is irreducible and $f \neq 0$, the $A$-submodule $f(M)$ of $V$ is isomorphic to $M$, and since $V$ is completely reducible $f(M)$ has a complement in $V$. Hence there exists $\theta \in \operatorname{Hom}_{A}(V, M)$ such that $\theta f$ is the identity on $M$. Now if $g \in \mathfrak{T}(M)$ is arbitrary then $e=g \theta \in E$ satisfies $e f=g$. It follows that $f$ generates $\tilde{T}(M)$ as $E$-module. Since this holds for any nonzero element $f \in \mathfrak{F}(M), \mathfrak{F}(M)$ is irreducible.

(1.4) LemMa. The algebra $E$ is semisimple.

Proof. Write $V \cong \sum n_{i} U_{i}$ (direct sum) where the $U_{i}$ are irreducible $A$-submodules of $V$ and the $n_{i}$ are positive integers. By (1.2) and (1.3) we have that $E \cong \sum n_{i} F\left(U_{i}\right)$; this is an expression of the left regular $E$-module ${ }_{E} E$ as a sum of irreducibles. By [6, Theorem 25.2], $E$ is semisimple.

(1.4)' Corollary. (i) $\mathfrak{F}^{\prime}$ is additive.

(ii) $\mathfrak{F}^{\prime}$ takes irreducibles to irreducibles. 
Proof. From (1.2)(i) and (ii) and (1.4) it is clear that the roles of $A$ and $E$ are symmetric; thus (1.4)' follows from (1.3) on replacing $A, E$ and $\mathfrak{F}$ by $E, A$ and $\mathfrak{F}^{\prime}$ respectively.

(1.4)" Corollary. (i) The functors $\mathfrak{F}$ and $\mathfrak{F}^{\prime}$ define mutually inverse bijections between the sets of equivalence classes of irreducible A-modules and irreducible E-modules.

(ii) For any $X \in \mathbb{Q}$, we have $\mathfrak{F}^{\prime} \mathfrak{F}(X) \cong X$ and similarly for $\mathfrak{F}^{\prime} \mathfrak{F}^{\prime}$.

Proof. The functorial nature of $\mathfrak{F}$, $\mathfrak{F}^{\prime}$ ensures that they define maps between equivalence classes of modules. If $M$ is an irreducible $A$-module, we have the evaluation map $\varepsilon: M \rightarrow \mathfrak{F}^{\prime} \mathfrak{F}(M)$ given by $m \mapsto \varepsilon_{m}$, where $\varepsilon_{m}(\phi)=\phi(m)(\phi \in$ $\mathfrak{F}(M)=\operatorname{Hom}_{A}(M, V)$ ). Clearly ker $\varepsilon=0$, and since $\mathfrak{F}^{\prime} \mathfrak{F}(M)$ is irreducible (by (1.3)(i) and $\left.(1.4)^{\prime}(\mathrm{i})\right)$ we have that $\varepsilon: M \rightarrow \mathfrak{F}^{\prime} \mathfrak{F}(M)$ is an isomorphism. Similarly, $N \cong \mathfrak{F} \mathfrak{F}^{\prime}(N)(N \in \mathcal{E})$ and (i) follows. Part (ii) is immediate by additivity.

(1.5) LeMma. There is a natural isomorphism of vector spaces $\operatorname{Hom}_{A}(N, M) \rightarrow$ $\operatorname{Hom}_{E}(\mathfrak{F}(M), \mathfrak{F}(N))(N, M \in \mathbb{Q})$, and similarly for $\mathfrak{F}^{\prime}$.

Proof. For $\phi \in \operatorname{Hom}_{A}(N, M)$, we have $\mathfrak{F}(\phi) \in \operatorname{Hom}_{E}(\mathfrak{F}(M)$, $\mathfrak{F}(N))$ given by composition with $\phi$ : $\mathfrak{F}(\phi)(\theta)=\theta \phi(\theta \in \mathfrak{F}(M))$. The map $\phi \mapsto \mathfrak{F}(\phi)$ is linear, and we have similarly a linear map $\sigma \mapsto F^{\prime}(\sigma): \operatorname{Hom}_{E}(\mathfrak{F}(M)$, F $(N)) \rightarrow$ $\operatorname{Hom}_{A}\left(\mathfrak{F}^{\prime} \mathfrak{F}(N), \mathfrak{F}^{\prime} \mathfrak{F}(N)\right)$. Now from $(1.4)^{\prime \prime}$, the evaluation maps $N \rightarrow \mathfrak{F}^{\prime} \mathfrak{F}(N)$ and $M \rightarrow \mathfrak{F}^{\prime} \mathfrak{F}(M)$ are isomorphisms, and it is easily checked that for $\phi \in \operatorname{Hom}_{A}(N, M)$ and $n \in N$, we have $\mathfrak{F}^{\prime} \mathfrak{F}(\phi)\left(\varepsilon_{n}\right)=\varepsilon_{\phi(n)}$. Hence $\mathfrak{F}(\phi)=\mathfrak{F}(\psi)$ implies that $\varepsilon_{\phi(n)}=$ $\varepsilon_{\psi(n)}$ for all $n \in N$, whence $\phi=\psi$. Thus $\phi \mapsto \mathfrak{F}(\phi)$ is an injective map between spaces of the same dimension. The result follows.

(1.5)' REMARK. This shows that $\mathfrak{F}, \mathfrak{F}^{\prime}$ are mutually adjoint functors

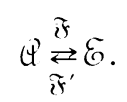

(1.6) Proposition. Let $A$ be a complex semisimple associative algebra as above and $V$ any (left) $A$-module (not assumed faithful). Let $E=\operatorname{Hom}_{A}(V, V)$. Then

(i) The functors $\mathfrak{F}$ and $\mathfrak{F}^{\prime}$ defined in (1.1) define inverse bijections between the equivalence classes of irreducible A-submodules of $V$ and irreducible E-modules.

(ii) The conclusion of (1.4)" remains true provided that we restrict $X \in Q$ to modules whose irreducible components occur in $V$.

(iii) The conclusion of (1.5) remains true provided that at least one of $N, M$ has only irreducible components occurring in $V$.

We now turn our attention to induced modules. Assume that $A$ is a finite-dimensional semisimple $\mathbf{C}$-algebra and that $B$ is a semisimple subalgebra which contains the identity element of $A$. Let $M$ be a left $B$-module.

(1.7) Definition. The $A$-module induced from $M$ is $M^{A}=\operatorname{Hom}_{B}(A, M)$ where the $A$ action is given by

$$
(\text { af })(x)=f(x a) \quad\left(a, x \in A, f \in M^{A}\right)
$$


REMARK. We shall be mostly interested in the case where $A$ is the group algebra (possibly twisted) of a finite group, and $B$ is the algebra of a subgroup. It is well known that in this case our definition and the alternative (dual): $M^{A}=A \otimes_{B} M$ yield isomorphic modules.

(1.8) LeMma. There is a natural surjective B-homomorphism $\pi: M^{A} \rightarrow M$.

Proof. Define $\pi f=f(1)\left(f \in M^{A}\right)$. It is easily checked that $\pi$ is a $B$-homomorphism. Since $B$ is semisimple, $B$ has a complement $B^{\prime}$ in $A$ (regarded as a $B$-module): $A=B \oplus B^{\prime}$ ( $B$-module decomposition). So for $m \in M$, there is $f \in M^{A}$ satisfying $f(b)=b m(b \in B)$ and $f\left(b^{\prime}\right)=0\left(b^{\prime} \in B^{\prime}\right)$. It follows that $\pi$ is surjective.

(1.9) Lemma. If $A, B$ and $C$ are semisimple C-algebras with $1_{A} \in C \subset B \subset A$ and $M$ is a left $C$-module then there is a canonical A-isomorphism: $\left(M^{B}\right)^{A} \cong M^{A}$.

The proof (which is standard) is omitted.

(1.10) Lemma. With $A, B, C$ and $M$ as in (1.9), restriction of homomorphisms is a $B$-module surjection: $M^{A} \rightarrow M^{B}$.

Proof. This follows readily from (1.8) and (1.9); the map $\pi$ of (1.8) (with $M^{B}$ replacing $M$ ) is identified in (1.9) as restriction of maps.

(1.11) Lemma. With $A, B$ and $M$ as in (1.7), let $E_{B}=\operatorname{End}_{B}(M)$ and let $E_{A}=$ End $_{A}\left(M^{A}\right)$. Then there is a canonical embedding of C-algebras: $E_{B} \hookrightarrow E_{A}$. The image of this map consists of $\left\{f \in E_{A} \mid f(\operatorname{ker} \pi) \subset \operatorname{ker} \pi\right\}(\pi$ as in (1.8)).

Proof. Let $e \in E_{B}$ and define $\tilde{e}: M^{A} \rightarrow M^{A}$ by $\tilde{e} \phi(a)=e(\phi(a))\left(\phi \in M^{A}\right.$, $a \in A$ ). It is easily verified that $\tilde{e} \in E_{A}$ and that $e \mapsto \tilde{e}$ is a $\mathrm{C}$-algebra homomorphism. If $\tilde{e}_{1}=\tilde{e}_{2}$, then $e_{1}(\phi(1))=e_{2}(\phi(1))$ for all $\phi \in M^{A}$, and since $\pi: \phi \mapsto \phi(1)$ is surjective (1.8), it follows that $e_{1}=e_{2}$. Thus $e \mapsto \tilde{e}$ is injective.

If $\phi(1)=0$, then $\tilde{e} \phi(1)=e(\phi(1))=0$; thus each element $\tilde{e}\left(e \in E_{B}\right)$ of $E_{A}$ preserves $\operatorname{ker} \pi$. Conversely, if $f \in E_{A}$ satisfies $f(\operatorname{ker} \pi) \subset \operatorname{ker} \pi$, then $f$ induces a $B$-endomorphism $e$ of $M^{A} / \operatorname{ker} \pi$, which is isomorphic as $B$-module (by (1.8)) to $M$. We then have (for $\left.\phi \in M^{A}\right), e(\pi \phi)=\pi(f \phi)$, and a short computation shows that $(\tilde{e} \phi)(a)=(f \phi)(a)\left(\phi \in M^{A}, a \in A\right)$. Thus $f=\tilde{e}$ as required.

Let $N$ be any $A$-module. Then $\operatorname{Hom}_{A}\left(N, M^{A}\right)$ is an $E_{A}$ module in the notation of (1.11), and by (1.11) may be regarded as an $E_{B}$-module by restriction. On the other hand $N$ is a $B$-module by restriction and thus $\operatorname{Hom}_{B}(N, M)$ is an $E_{B}$-module in the obvious way.

(1.12) Proposition (Strong Frobenius Reciprocity). In the above notation, there is a natural $E_{B}$-isomorphism: $\operatorname{Hom}_{B}(N, M) \rightarrow \operatorname{Hom}_{A}\left(N, M^{A}\right)$.

Proof. If $f \in \operatorname{Hom}_{B}(N, M)$, define $\tilde{f}: N \rightarrow M^{A}$ by $[\tilde{f}(n)](a)=f(a n)(n \in N$, $a \in A)$.

Then $\tilde{f} \in \operatorname{Hom}_{A}\left(N, M^{A}\right)$, since $[\tilde{f}(a n)]\left(a^{\prime}\right)=f\left(a^{\prime} a n\right)=[\tilde{f}(n)]\left(a^{\prime} a\right)=$ $[a(\tilde{f}(n))]\left(a^{\prime}\right)$. Conversely, if $g \in \operatorname{Hom}\left(N, M^{A}\right)$, then $\pi g \in \operatorname{Hom}_{B}(N, M)$, and it is easily checked that $g \mapsto \pi g$ is inverse to $f \mapsto \tilde{f}$. 
It remains to check that this isomorphism commutes with the action of $E_{B}$. For this, let $e \in E_{B}$ and take $f \in \operatorname{Hom}_{B}(N, M)$. We have to show that $(e \tilde{f})=\tilde{e} \tilde{f}$, i.e. that for all $n \in N$ and $a \in A$, we have $[(e \tilde{f})(n)](a)=[\tilde{e}(\tilde{f}(n))](a)$. This latter equation is quickly verified by a check of the definitions.

(1.13) Theorem. With $A, B, M, E_{A}$ and $E_{B}$ as above, let $\mathfrak{F}_{A}$ and $\mathfrak{F}_{B}$ be the functors of Definition (1.1) arising respectively from the $A$-module $M^{A}$ and $B$-module $M$. Let $X$ be any $B$-submodule of $M$ and $Y$ any $A$-module. Then there is a linear isomorphism

$$
\operatorname{Hom}_{B}(X, Y) \stackrel{\cong}{\rightarrow} \operatorname{Hom}_{E_{B}}\left(\mathfrak{F}_{B}(X), \mathfrak{F}_{A}(Y)\right),
$$

where $Y$ is thought of as a $B$-module by restriction, and $\mathfrak{F}_{A}(Y)$ is thought of as an $E_{B}$-module via the canonical embedding of (1.11).

Proof. Since $B$ is semisimple, $\operatorname{Hom}_{B}(X, Y) \cong \operatorname{Hom}_{B}(Y, X)$ as vector spaces. By (1.5) and (1.6) we have

$$
\operatorname{Hom}_{B}(Y, X) \cong \operatorname{Hom}_{E_{B}}\left(\mathfrak{\mho}_{B}(X), \mathfrak{\mho}_{B}(Y)\right) .
$$

But by (1.12) we have an $E_{B}$-isomorphism

$$
\mathfrak{F}_{B}(Y)=\operatorname{Hom}_{B}(Y, M) \stackrel{\cong}{\rightarrow} \operatorname{Hom}_{A}\left(Y, M^{A}\right)=\mathfrak{F}_{A}(Y),
$$

whence the result.

REMARK. Clearly (1.13) is essentially a restatement of the "strong Frobenius reciprocity" result (1.12). It may be worth noting that (1.12) may also be expressed in the language of (1.13) as follows: let $\mathcal{Q}, \mathfrak{B}, \mathcal{E}_{A}$ and $\mathcal{E}_{B}$ be the categories of left $A, B, E_{A}$ and $E_{B}$-modules respectively. Then we have the following commutative diagram of functors:

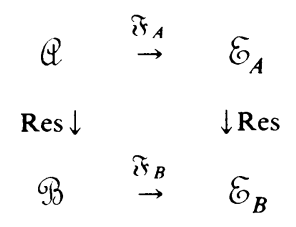

Of course various other commutative diagrams may be constructed using the adjoints $\mathfrak{F}_{A}^{\prime}$, Ind, etc. of the above functors.

We shall apply (1.13) to study induced characters of finite groups, and reformulate the results accordingly. If $H$ is a subgroup of the finite group $G, M$ a $\mathrm{C} H$-module and $\chi$ the character of $M$, we write $\operatorname{Ind}(H \rightarrow G ; \chi)$ for the character of $M^{\mathrm{C} G}$. Similarly we write $\operatorname{Res}(G \rightarrow H ; \phi)$ for the restriction to $H$ of a character $\phi$ of $G$.

Let $G, H, M, \chi$ be as in the previous paragraph. For any intermediate subgroup $K,(H \leqslant K \leqslant G)$, let $E_{K}=$ End $_{\mathrm{C} K}\left(M^{\mathrm{C} K}\right)$ and let $\mathfrak{F}_{K}$ be the functor associated with the $\mathbf{C} K$-module $M^{\mathbf{C} K}$. If $X$ is a $\mathbf{C} K$-module with character $\eta$, we write $\eta^{\prime}$ for the character of the $E_{K}$-module $\mathfrak{F}_{K}(X)$. By (1.4), if $\eta$ is an irreducible constituent of $\operatorname{Ind}(H \rightarrow K ; \chi)$ then $\eta^{\prime}$ is an irreducible character of $E_{K}$. By (1.11) $E_{K}$ embeds in $E_{G}$ and so characters of $E_{G}$ yield characters of $E_{K}$ by restriction. 
(1.14) Corollary. Suppose (as above) that $H \leqslant K \leqslant G$ are finite groups and that $\chi$ is a complex character of $H$. Let $E_{K} \subset E_{G}$ be the endomorphism algebras associated with the characters $\operatorname{Ind}(H \rightarrow K ; \chi)$ and $\operatorname{Ind}(H \rightarrow G ; \chi)$. Then

(i) The map $\eta \mapsto \eta^{\prime}$ defines a bijection from the set of irreducible constituents of Ind $(H \rightarrow K ; \chi)$ to the set of irreducible characters of $E_{K}$.

(ii) If $\zeta$ is any character of $G$ and $\zeta^{\prime}$ is the corresponding character of $E_{G}$, then the multiplicity of $\eta$ in $\operatorname{Res}(G \rightarrow K ; \zeta)$ is equal to the multiplicity of $\eta^{\prime}$ in $\operatorname{Res}\left(E_{G} \rightarrow E_{K} ; \zeta^{\prime}\right)$ $\left(\eta, \eta^{\prime}\right.$ as in (i)).

Proof. That $\eta \mapsto \eta^{\prime}$ is a bijection follows from (1.4) and (1.6). Let $Y$ be a $\mathbf{C} G$ module with character $\zeta$. The multiplicity of $\eta$ in $\operatorname{Res}(G \rightarrow K$; $\zeta)$ is $\operatorname{dim}_{\mathbf{C}}\left(\operatorname{Hom}_{\mathbf{C} K}(X, Y)\right)$, which by (1.13) equals $\operatorname{dim}_{\mathbf{C}}\left(\operatorname{Hom}_{E_{K}}\left(\mathfrak{F}_{K}(X), \mathfrak{F}_{K}(Y)\right)\right.$. But as $E_{K}$-module, $\mathfrak{F}_{G}(Y)$ has character $\operatorname{Res}\left(E_{G} \rightarrow E_{K} ; \zeta^{\prime}\right)$ while $\mathscr{F}_{K}(X)$ is irreducible with character $\eta^{\prime}$. Thus the multiplicity of $\eta^{\prime}$ in $\operatorname{Res}\left(E_{G} \rightarrow E_{K} ; \zeta^{\prime}\right)$ equals the dimension of $\operatorname{Hom}_{E_{K}}\left(\mathscr{F}_{K}(X), \mathscr{F}_{K}(Y)\right)$, and (ii) follows.

Our final result of this section concerns the endomorphism algebras of isomorphic modules.

(1.15) Proposition. Let $A$ be as in (1.1) and $\sigma: V_{1} \rightarrow V_{2}$ an isomorphism of $A$-modules. Let $E_{i}=\operatorname{End}_{A}\left(V_{i}\right)(i=1,2)$, and for any $A$-module $M$ with character $\zeta$, let $\zeta_{i}^{\prime}$ be character of the associated $E_{i}$-module $\mathfrak{F}_{i}(M)=\operatorname{Hom}_{A}\left(M, V_{i}\right)$. Then $e \mapsto \sigma e \sigma^{-1}$ is an isomorphism $E_{1} \rightarrow E_{2}$, and $\zeta_{1}^{\prime}(e)=\zeta_{2}^{\prime}\left(\sigma e \sigma^{-1}\right)$ for all $e \in E_{1}$.

Proof. It is obvious that $e \mapsto \sigma e \sigma^{-1}$ is an isomorphism: $E_{1} \rightarrow E_{2}$. Also, $\theta: f \mapsto \sigma f$ is a vector space isomorphism $\mathfrak{F}_{1}(M) \rightarrow \mathfrak{F}_{2}(M)$ and we have for $e \in E_{1}$ the following commutative diagram:

$$
\begin{array}{ccc}
\mathfrak{F}_{1}(M) & \stackrel{\theta}{\rightarrow} & \mathfrak{F}_{2}(M) \\
e \downarrow & & \downarrow \sigma e \sigma_{1}^{-1} \\
\mathfrak{F}_{1}(M) & \stackrel{\theta}{\rightarrow} & \mathfrak{F}_{1}(M)
\end{array}
$$

It follows that the trace of $E$ on $\mathfrak{F}_{1}(M)$ equals the trace of $\sigma e \sigma^{-1}$ on $\mathfrak{F}_{2}(M)$, i.e. that $\zeta_{1}^{\prime}(e)=\zeta_{2}^{\prime}\left(\sigma e \sigma^{-1}\right)$.

2. Characters of projective representations. In this section we introduce some notation and state without proof some straightforward results concerning projective representations of finite groups. In what follows $\mu$ will be a fixed 2-cocycle for the finite group $G$. That is, $\mu: G \times G \rightarrow \mathbf{C}^{\times}$satisfies

$$
\mu(x, y) \mu(x y, z)=\mu(x, y z) \mu(y, z) \quad(x, y, z \in G) .
$$

Let $(\mathbf{C} G)_{\mu}$ be the twisted group algebra of $G$ determined by $\mu ;(\mathbf{C} G)_{\mu}$ has linear basis $\{\bar{x} \mid x \in G\}$ and multiplication satisfying $\bar{x} \bar{y}=\mu(x, y) \overline{x y}(x, y \in G)$. If $R$ is a projective representation of $G$ whose 2-cocycle is $\mu$ (i.e. $R(x) R(y)=\mu(x, y) R(x y)$ for $x, y \in G$-we call such representations $\mu$-representations), then $R$ is associated in an obvious way with a representation of $(\mathrm{C} G)_{\mu}$; conversely, a representation of $(\mathrm{C} G)_{\mu}$ yields a projective representation of $G$, with 2-cocyle $\mu$. 
Define the character of a projective representation $R$ to be $\rho: G \rightarrow \mathbf{C}$, given by $\rho(x)=\operatorname{trace} R(x)(x \in G)$. We say that $\rho$ is a $\mu$-character of $G$ if $R$ is a $\mu$-representation. Let $\operatorname{Irr}_{\mu}(G)$ be the set of all irreducible $\mu$-characters of $G$, and $\operatorname{Char}_{\mu}(G)$ the $\mathbf{Z}$-module of complex functions on $G$ which is generated by the $\mu$-characters. For $\rho \in \operatorname{Char}_{\mu}(G)$ define $\check{\rho}: G \rightarrow \mathrm{C}$ by

$$
\check{\rho}(x)=\left[\mu\left(x, x^{-1}\right) \mu(1, x)\right]^{-1} \rho\left(x^{-1}\right) \quad(x \in G) .
$$

Then $\rho(x)=\operatorname{trace} R(x)^{-1}$. For $\rho, \sigma \in \operatorname{Char}_{\mu}(G)$ define their inner product to be

$$
(\rho, \sigma)=|G|^{-1} \sum_{x \in G} \rho(x) \check{\sigma}(x) \text {. }
$$

We define induced characters as follows: let $\sigma \in \operatorname{Char}_{\mu}(H)$, where $H \leqslant G$ and $\mu$ is also used to denote the restriction of $\mu$ to $H$. Define $\operatorname{Ind}(H \rightarrow \mathrm{G} ; \sigma)$ by

$$
\operatorname{Ind}(H \rightarrow G ; \sigma)(x)=|H|^{-1} \sum_{y} \frac{u(x, y)}{\mu\left(y, y^{-1} x y\right)} \sigma\left(y^{-1} x y\right)
$$

(for $x \in G$; the summation extends over the elements $y \in G$ with $y^{-1} x y \in H$ ).

(2.1) LEMMA. (i) $\operatorname{Irr}_{\mu}(G)$ is finite.

(ii) Let $\rho, \sigma \in \operatorname{Irr}_{\mu}(G)$. Then

$$
(\rho, \sigma)= \begin{cases}1 & \text { if } \rho=\sigma \\ 0 & \text { otherwise } .\end{cases}
$$

(iii) Let $H \leqslant G$ and suppose $\sigma \in \operatorname{Char}_{\mu}(H)$. Then $\operatorname{Ind}(H \rightarrow G$; $\sigma)$ is a $\mu$-character of $G$. Indeed, if $M$ is a $(\mathrm{CH})_{\mu}$-module corresponding to $\sigma$, then the $(\mathrm{C} G)_{\mu}$-module induced from $M$ corresponds to $\operatorname{Ind}(H \rightarrow G ; \sigma)$.

(iv) If $\sigma \in \operatorname{Char}_{\mu}(G)$ and $H \leqslant G$, then

$$
\operatorname{Ind}(H \rightarrow G ; \operatorname{Res}(G \rightarrow H ; \sigma))=\sigma \cdot \operatorname{Ind}(H \rightarrow G ; 1)
$$

(where the latter Ind is ordinary induction of characters).

(v) Let $H \leqslant G, \rho \in \operatorname{Char}_{\mu}(G)$ and $\sigma \in \operatorname{Char}_{\mu}(H)$. Then

$$
(\rho, \operatorname{Ind}(H \rightarrow G ; \sigma))=(\operatorname{Res}(G \rightarrow H ; \rho), \sigma),
$$

where inner products in $\mathrm{Char}_{\mu}(H)$ are defined in analogous fashion to inner products in $\operatorname{Char}_{\mu}(G)$.

Lemma (2.1) may be proved by using $\mu$ to construct a central extension $\tilde{G}$ of $G$ and then using the corresponding results for the ordinary representation theory of $\tilde{G}$. Alternatively, one may proceed directly, imitating the proof of these results.

If $G$ is a subgroup of $G_{0}$ then for $x \in G_{0}$ we define the 2-cocycle $x \mu$ of the group ${ }^{x} G=x G x^{-1}$ by

$$
(x \mu)(y, z)=\mu\left(y^{x}, z^{x}\right) \quad\left(y, z \in{ }^{x} G\right) .
$$

If $\rho \in \operatorname{Char}_{\mu}(G)$ then $x \rho: h \mapsto\left(h^{x}\right)$ is an $x \mu$-character of ${ }^{x} G\left(h \in{ }^{x} G\right)$. If $x$ above is in $G$, then $x \mu$ and $\mu$ are cohomologous, i.e. there is a function $\alpha$ : $G \rightarrow \mathbf{C}^{\times}$such that

$$
(x \mu)(y, z)=\alpha(y) \alpha(z) \alpha(y z)^{-1} \mu(y, z) \text { for } y, z \in G \text {. }
$$


Indeed, $\alpha(g)=\mu(g, x)^{-1} \mu\left(x, g^{x}\right)$ is such a function. If $H \leqslant G$ and $x \in G$ then for any $\mu$-character $\rho$ of $H$, the function $\bar{x} \rho$ defined by

$$
(\bar{x} \rho)(h)=\frac{\mu(h, x)}{\mu\left(x, h^{x}\right)} \rho\left(h^{x}\right)
$$

is a $\mu$-character of ${ }^{x} H$.

The following two results are easily verified:

(2.2) Lemma. If $\rho$ is a $\mu$-character of $G$ and $\alpha: G \rightarrow \mathbf{C}^{x}$ is any function, then the product $\alpha \rho$ is a $\mu^{\prime}$-character of $G$, where $\mu^{\prime}(x, y)=\alpha(x) \alpha(y) \alpha(x y)^{-1} \mu(x, y)$. Moreover $\rho \mapsto \alpha \rho$ is a bijection from $\operatorname{Irr}_{\mu}(G)$ to $\operatorname{Irr}_{\mu^{\prime}}(G)$.

(2.3) LEMMA. If $H \leqslant G$ and $x, y \in G$ then for any $\mu$-character $\rho$ of $H$ we have

(a) $\bar{x}(\bar{y} \rho)=\overline{x y} \rho$.

(b) $\operatorname{Ind}(H \rightarrow G ; \rho)=\operatorname{Ind}\left({ }^{x} H \rightarrow G, \bar{x} \rho\right)$.

3. Endomorphism algebras of induced cuspidal representations. This section contains a résumé and slight extension of some of the results of [9]. These will be used later in conjunction with the results of $\S 1$ to establish the comparison theorem $(\$ 5$ below). In addition, the results of $\$ 4$ concerning generic algebras and their specializations depend on the facts recalled here.

We shall adhere closely to the notation of [9]. With $G$ and $W$ as in the introduction, let $E$ be the Euclidean space on which $W$ acts, $\Sigma \subset E$ the corresponding root system, $\Pi \subset \Sigma$ the set of simple roots determined by the choice of a fixed Borel subgroup $B \subset G$. Then the standard parabolic subgroups of $G$ are the groups $P_{J}, J \subset \Pi$. For $w \in W=N / B \cap N$, denote by $\dot{w}$ a (fixed) representative of $w$ in $N$. If $R$ is a (complex) representation of a subgroup $H$ of $G$ and $x$ is an element of $G$, then $x R$ is defined as the representation of ${ }^{x} H$ given by $(x R)(g)=R\left(g^{x}\right)$, for $g \in{ }^{x} H$. We use $\tilde{D}$ to denote the representation of $P_{L}(L \subset \Pi)$ obtained by lifting the representation $D$ of $M_{L}$ (contrary to [9], where $D^{*}$ was used) and we use " " " also to denote other liftings of representations and characters via projections such as $P_{L} \rightarrow M_{L}$, relying on the context to make it clear what is the kernel of the projection involved. Following Curtis [3] we make the following definition for $\xi \in \operatorname{Char}(G)$ :

(3.1) Definition. The truncation $\xi_{\left(P_{J}\right)}$ of $\xi$ from $G$ to $P_{J}(J \subset \Pi)$ is given by

$$
\xi_{\left(P_{J}\right)}=\sum_{n}\left(\operatorname{Res}\left(G \rightarrow P_{J} ; \xi\right), \tilde{\eta}\right) \tilde{\eta}
$$

the sum being over $\left\{\eta \in \operatorname{Irr}\left(M_{J}\right)\right\}$.

If $\xi$ is the character of a $G$-module $X$ then clearly $\xi_{\left(P_{J}\right)}$ is the character of the $P_{J}$-submodule of $X$ consisting of the $U_{J}$-invariant elements of $X$. The character $\xi$ of $G$ is called cuspidal if $\xi_{\left(P_{J}\right)}=0$ for each $J \subset \Pi, \mathrm{J} \neq \Pi$. The Harish-Chandra principle for $G$ implies that:

(3.2) Each $\xi \in \operatorname{Irr}(G)$ is a constituent of a unique character of the form $\operatorname{Ind}\left(P_{L} \rightarrow G ; \tilde{\delta}\right)$ where $\sigma$ is an irreducible cuspidal character of $M_{L}$, some $L \subset \Pi$.

Thus in particular if $L, L^{\prime} \subset \Pi$ and $\delta, \delta^{\prime}$ are irreducible cuspidal characters in $M_{L}, M_{L^{\prime}}$ respectively, then $\operatorname{Ind}\left(P_{L} \rightarrow G ; \tilde{\delta}\right)$ and $\operatorname{Ind}\left(P_{L^{\prime}} \rightarrow G, \tilde{\delta^{\prime}}\right)$ either coincide or 
are disjoint. It may further be shown that:

(3.3) The above induced characters are equal if and only if there exists $w \in W$ such that ${ }^{w} M_{L}=M_{L^{\prime}}$ and $w \delta=\delta^{\prime}$.

The irreducible characters of $G$ may therefore be described in terms of the decomposition of induced cuspidal characters. A key concept in this decomposition is that of the ramification group:

(3.4) Definition. Let $\delta$ be an irreducible cuspidal character of $M_{L}(L \subset \Pi)$. The ramification group $W(\delta)$ of $\delta$ is defined by

$$
W(\delta)=\{w \in W \mid w L=L, w \delta=\delta\} .
$$

For the remainder of this work we take $L \subset \Pi$ and the cuspidal irreducible character $\delta$ of $M_{L}$ to be fixed; we also fix a representation $D$ of $M_{L}$ with character $\delta$.

Let $M^{0}$ be the subgroup of $G$ generated by $M_{L}$ and the elements $\{\dot{w} \mid w \in W(\delta)\}$. Then $M_{L} \unlhd M^{0}$ and $M^{0} / M_{L} \cong W(\delta)$. In particular any 2-cocycle $\mu$ of $W(\delta)$ becomes a 2-cocycle of $M^{0}$ by the rule

$$
\mu\left(\dot{w}_{1} m_{1}, \dot{w}_{2} m_{2}\right)=\mu\left(w_{1}, w_{2}\right) \quad\left(m_{i} \in M_{L}, w_{i} \in W(\delta)\right) .
$$

(3.5) Proposition (cf. $[9,(3.6)])$. The representation $D$ of $M_{l}$ extends to a projective representation $D^{0}$ of $M^{0}$ associated with a 2-cocycle $\mu$ of $W(\delta)$. The cohomology class of $\mu$ is uniquely determined by $\delta$.

In (3.10) below a fixed choice of $\mu$ shall be made.

(3.6) Definition. Let $\Gamma$ be the set of roots $a \in \Sigma, a \notin L$ satisfying the following:

(i) $w(L \cup\{a\}) \subset \Pi$ for some $w \in W$.

(ii) $v(a, L)^{2}=1$ (equivalently $v(a, L)(L)=L$ - see $[9,(2.4)]$ ).

(iii) $v(a, L) \in W(\delta)$.

(iv) With $w$ as in (i) and $K=w(L \cup\{a\})$, the character $\operatorname{Ind}\left(P_{w L} \cap M_{K} \rightarrow M_{K} ; w \tilde{\delta}\right)$ has exactly two irreducible constituents (this is always true) whose degrees are not equal.

Note that this notation is essentially the same as in [9]- see (3.2), (2.4) of [9].

(3.7) Definition. For $a \in \Gamma$, let $p_{a}>1$ be the ratio of the degrees of the characters mentioned in (3.6) (iv) above.

By $[9,(3.18)] p_{a}$ is an integral power of $p$, and by [loc. cit (3.20)] $p_{a}$ is independent of $w$.

(3.8) Lemma. Suppose that $L_{1} \subset \Pi$ and $\delta_{1}$ is a cuspidal character of $M_{L_{1}}$. Let $\Gamma_{1}$ and $p_{a}^{\prime}\left(a \in \Gamma_{1}\right)$ be defined for $\left(L_{1}, \delta_{1}\right)$ in the same way as $\Gamma$ and $p_{a}(a \in \Gamma)$ are defined for $(L, \delta)$. If $v \in W$ satisfies $v L=L_{1}$ and $v \delta=\delta_{1}$ then we have $v \Gamma=\Gamma_{1}$ and $p_{v a}^{\prime}=p_{a}$ for all $a \in \Gamma$.

Proof. Let $a \in \Gamma_{1}$. Choose $w \in W$ satisfying (3.6)(i); then let $K=w(L \cup\{a\})$ as in (3.6)(iv) and let $w_{1}=w v$. Then from (3.6)(i)-(iv) respectively, we have

(i) $w_{1}\left(L_{1} \cup\{v a\}\right)=w(L \cup\{a\}) \subset \Pi$,

(ii) $v\left(v a, L_{1}\right)^{2}=\left(v \cdot v(a, L) \cdot v^{-1}\right)^{2}=1$,

(iii) $v\left(v a, L_{1}\right) \in v W(\delta) v^{-1}=W\left(\delta_{1}\right)$ and,

(iv) if $K_{1}=w_{1}\left(L_{1} \cup\{v a\}\right)$ then $K_{1}=K$ and $\operatorname{Ind}\left(P_{w_{1} L_{1}} \cap M_{K_{1}} \rightarrow M_{K_{1}} ; w_{1} \delta_{1}\right)=$ Ind $\left(P_{w L} \cap M_{K} \rightarrow M_{K} ; w \delta\right)$ has two constituents whose degrees are not equal. 
Hence $v a \in \Gamma_{1}$, so that $v \Gamma \subset \Gamma_{1}$. Similarly $v^{-1} \Gamma_{1} \subset \Gamma$. The equality of the induced representations in (iv) above shows that $p_{v a}^{\prime}=p_{a}$ for all $a \in \Gamma$.

Write $\Sigma^{+}$for the set of positive roots and $\Gamma^{+}=\Gamma \cap \Sigma^{+}$. For $w \in W$ write $N(w)=\left\{a \in \Sigma^{+} \mid w a \neq \Sigma^{+}\right\}$. Lemma (2.7) of [9] yields

(3.9) Lemma. The group $R(\delta)$ generated by $\{v(a, L) \mid a \in \Gamma\}$ is a normal subgroup of $W(\delta)$ and acts as a reflection group on $L^{\perp}$. The projection to $L^{\perp}$ of $\Gamma$ is a root system for $R(\delta)$; the projection of $\Gamma^{+}$is a positive system and the projection of $\Delta=\left\{a \in \Gamma^{+} \mid N(v(a, L)) \cap \Gamma=\{a\}\right\}$ the corresponding fundamental system. Moreover, $W(\delta)$ is a semidirect product $R(\delta) \cdot C(\delta)$ where $C(\delta)=\left\{w \in W(\delta) \mid w \Gamma^{+}=\right.$ $\left.\Gamma^{+}\right\}$.

Let $V$ be a $\mathbf{C} P_{L}$-module realizing the representation $D$ and write

$$
\mathscr{F}_{G}(D)=\left\{f: G \rightarrow V \mid f(x y)=\tilde{D}(x) f(y) \text { for all } x \in P_{L}, y \in G\right\} .
$$

Clearly, $\mathscr{F}_{G}(D)$ is naturally isomorphic to the $\mathbf{C} G$-module $\operatorname{Hom}_{\mathbf{C} P_{l}}(\mathbf{C} G, V)$ realizing the representation $\operatorname{Ind}\left(P_{L} \rightarrow G ; \tilde{D}\right)$.

Let $E_{G}(D)=\operatorname{End}_{C G}\left(\mathscr{F}_{G}(D)\right)$. We can now state the main theorem (Theorem (4.14)) of [9]:

(3.10) Theorem. The algebra $E_{G}(D)$ has a C-basis $\left\{T_{w} \mid w \in W(\delta)\right\}$ whose multiplication table satisfies (i) to (iv) below for all $w \in W(\delta), x \in C(\delta)$ and $a \in \Delta$. In (iii) and (iv) $v=v(a, L)$.

$$
\begin{gathered}
T_{w} T_{x}=\mu(w, x) T_{w x}, \\
T_{x} T_{w}=\mu(x, w) T_{x w}, \\
T_{v} T_{w}= \begin{cases}T_{v w} & \text { if } \left.w^{-1} a \in \Gamma^{+}\right), \\
p_{a} T_{v w}+\left(p_{a}-1\right) T_{w} & \text { if } \left.w^{-1} a \notin \Gamma^{+}\right),\end{cases} \\
T_{w} T_{v}= \begin{cases}T_{w v} & \text { if } \left.w a \in \Gamma^{+}\right), \\
p_{a} T_{w v}+\left(p_{a}-1\right) T_{w} & \text { if } \left.w a \notin \Gamma^{+}\right) .\end{cases}
\end{gathered}
$$

The 2-cocycle $\mu$ of $W(\delta)$ appearing in (i) and (ii) also satisfies (3.5) above, and has the further properties that $\mu(x v, y w)=\mu(x, y)$ for all $x, y \in C(\delta), v, w \in R(\delta)$, and $\mu(x, 1)=\mu(1, x)=1$ for all $x \in C(\delta)$.

Henceforth we assume that, in addition to $L$ and $D$, the cocycle $\mu$ is fixed in accordance with (3.10).

Apart from the slight complication due to the cocycle $\mu$ the algebra $E_{G}(D)$ has the same structure as the algebras arising from generalized Tits systems considered in [11].

In the course of proving our Comparison Theorem we will need to investigate $E_{G}(\dot{w} D)=\operatorname{End}_{\mathrm{C} G}\left(\operatorname{Ind}\left(P_{w L} \rightarrow G ; \widetilde{w} D\right)\right)$ for various $w \in W$ with $w L \subset \Pi$ as well as $E_{J}(\dot{w} D)=\operatorname{End}_{\mathrm{C}_{J}}\left(\operatorname{Ind}\left(P_{w L} \rightarrow P_{J} ; \overparen{\dot{w} D}\right)\right)$ for various $J$. For this we need some information on the structure of the groups $W_{J}^{w} \cap W(\delta)$, a description of the injection $E_{J}(\dot{w} D) \rightarrow E_{G}(\dot{w} D)$ (guaranteed by (1.11)) in terms of natural bases for these algebras, and a slight generalization of [9, Lemma (4.2)] to deal with homomorphisms between the spaces $\mathscr{F}_{G}(w D)$ for various $w$. 
(3.11) Definition. Write $V_{\delta}=\left\{w \in W \mid w L \subset \Pi, w \Gamma^{+} \subset \Sigma^{+}\right\}$.

(3.12) Lemma. Suppose $w L \subset \Pi$. Then $w W(\delta)$ contains an element of $V_{\delta}$.

Proof. Let $\Gamma_{1}$ be defined for $(w L, w \delta)$ in the same way as $\Gamma$ is defined for $(L, \delta)$. For all $v \in w W(\delta)$ we have $(v L, v \delta)=(w L, w \delta)$, so that by Lemma (3.8) $v \Gamma=\Gamma_{1}$, and hence $|N(v) \cap \Gamma|$ is the number of $a \in \Gamma^{+}$with $v a \in \Gamma_{1}^{-}$. Choose $v$ to minimize this number, and suppose that $v \Gamma^{+} \neq \Gamma_{1}^{+}$.

Since $\left|\Gamma^{+}\right|=\left|\Gamma_{1}^{+}\right|$there exists $a \in \Gamma^{+}$with va negative. By (3.9) (writing $\langle X\rangle$ for the linear span of any subset $X \subset E)$, we have $a \equiv \sum_{b \in \Delta} \lambda_{b} b(\bmod \langle L\rangle)$ for some $\lambda_{b} \geqslant 0$ and this gives $v a \equiv \sum_{b \in \Delta} \lambda_{b} v b(\bmod \langle w L\rangle)$. Since $v a$ is negative and not in $\langle w L\rangle$ it follows that $v b$ is negative for some $b \in \Delta$. Put $v^{\prime}=v v(b, L) \in w W(\delta)$.

Since $v(b, L)$ acts as a fundamental reflection in $R(\delta)$ it permutes $\Gamma^{+}-\{b\}$ and (modulo $\langle L\rangle$ ) takes $b$ to $-b$. We deduce that $v^{\prime}\left(\Gamma^{+}-\{b\}\right)=v\left(\Gamma^{+}-\{b\}\right)$ and $v^{\prime} b \equiv-v b(\bmod \langle w L\rangle)$, whence $\left|N\left(v^{\prime}\right) \cap \Gamma^{+}\right|=\left|N(v) \cap \Gamma^{+}\right|-1$. This contradicts the choice of $v$ and completes the proof.

(3.13) Lemma. Suppose $w \in V_{\delta}, w L \subset J \subset \Pi$. Then

$$
W_{J}^{w} \cap W(\delta)=R(\delta)_{\Lambda} \cdot\left(W_{J}^{w} \cap C(\delta)\right)
$$

where $\Lambda=\{a \in \Delta \mid w a \in\langle J\rangle\}$ and $R(\delta)_{\Lambda}$ is the parabolic subgroup of $R(\delta)$ generated by $\{v(a, L) \mid a \in \Lambda\}$.

Proof. By (3.9) we have that $W(\delta)=R(\delta) \cdot C(\delta)$ and $R(\delta)$ is generated by $\{v(a, L) \mid a \in \Delta\}$. By (3.9) with $W$ replaced by $W_{J}$ and $(L, \delta)$ replaced by $(w L, w \delta)$ we have that $W(w \delta) \cap W_{J}=R_{J}(w \delta) \cdot C_{J}(w \delta)$ where $R_{J}(w \delta)$ is generated by the set

$$
\left\{v(a, w L) \mid a \in \Gamma_{1} \cap\langle J\rangle \text { and } N(v(a, w L)) \cap \Gamma_{1}=\{a\}\right\}
$$

and

$$
C_{J}(w \delta)=\left\{x \in W(w \delta) \cap W_{J} \mid x\left(\Gamma_{1}^{+} \cap\langle J\rangle\right)=\Gamma_{1}^{+} \cap\langle J\rangle\right\}
$$

(where $\Gamma_{1}$ is as in (3.12) above).

If $x \in C_{J}(w \delta)$ then in particular $x \in W_{J}$, and so $N(x) \subset\langle J\rangle$. Moreover, by the definition of $C_{J}(w \delta), N(x) \cap\langle J\rangle \cap \Gamma_{1}^{+}=\varnothing$. Hence $N(x) \cap \Gamma_{1}^{+}=\varnothing$, and it follows that $x \Gamma_{1}^{+}=\Gamma_{1}^{+}$. Since $w \in V_{\delta}$ we have $\Gamma_{1}^{+}=w \Gamma^{+}$, and we deduce that $w^{-1} x w \Gamma^{+}=\Gamma^{+}$. Hence

$$
w^{-1} C_{J}(w \delta) w \leqslant C(\delta) \cap W_{J}^{w^{\prime}} .
$$

From $\Gamma_{1}^{+}=w \Gamma^{+}$we deduce also that for all $v \in W(w \delta)$ the map $a \mapsto w^{-1} a$ is a bijection from $N(v) \cap \Gamma_{1}^{+}$to $N\left(v^{w}\right) \cap \Gamma^{+}$. So if $a \in \Gamma_{1} \cap\langle J\rangle$ and $N(v(a, w L)) \cap$ $\Gamma_{1}=\{a\}$, then $w^{-1} a \in \Gamma$ and $N\left(v\left(w^{-1} a, L\right)\right) \cap \Gamma=\left\{w^{-1} a\right\}$. Thus $w^{-1} a \in \Delta$, and (since $a \in\langle J\rangle) w^{-1} a \in \Lambda$. Hence

$$
w^{-1} R_{J}(w \delta) w \leqslant R(\delta)_{\Lambda} .
$$

Now by (1) and (2),

$$
W_{J}^{w} \cap W(\delta)=w^{-1}\left(W_{J} \cap W(w \delta)\right) w \leqslant R(\delta)_{\Lambda} \cdot\left(W_{J}^{w} \cap C(\delta)\right) .
$$

The reverse inclusion is obvious. 
If $w \in W$ and $w L \subset \Pi$ then as in (3.10) of [9] we define $B_{D, \dot{w}}: \mathscr{F}_{G}(D) \rightarrow \mathscr{F}_{G}(\dot{w} D)$ by

$$
\left(B_{D, \dot{w}} f\right)(x)=\left|U_{w L}\right|^{-1} \sum_{y \in U_{w l .}} f\left(\dot{w}^{-1} y x\right) .
$$

From (3.11)(i) and (3.17) of [9] we have that $B_{D, \dot{w}}$ is a $C G$-isomorphism. The next result, which together with its proof is almost identical with Lemma (4.2) of [9] deals with composites of maps like $B_{D, \dot{w}}$.

(3.14) Lemma. Suppose $v, w \in W, v L \subset \Pi$, wv $w \subset \Pi, \Gamma_{1}=v \Gamma$. If $v^{-1} a \in \Sigma^{+}$for all $a \in \Gamma_{1} \cap N(w)$ (that is, $\left.N\left(v^{-1}\right) \cap N(w) \cap \Gamma_{1}=\varnothing\right)$ then

$$
B_{\dot{v} D, \dot{w}} B_{D, \dot{v}}=\left[(\text { ind } w)^{-1}(\text { ind } v)^{-1}(\text { ind } w v)\right]^{1 / 2} B_{D, \dot{w} v} .
$$

Because of its similarity with the proof of Lemma (4.2) of [9] which deals with the special case $v \in W(D)$, we omit the proof.

By (3.11) and (4.11) of [9] the elements $T_{v}(v \in W(\delta))$ of (3.10) are related to the $B_{D, \dot{\varepsilon}}$ by

$$
T_{v}=\varepsilon_{v}\left(p_{v} \text { ind } v\right)^{1 / 2} D^{0}(\dot{v}) B_{D, \dot{v}},
$$

where $D^{0}$ is as in (3.5) and $\varepsilon_{v}, p_{v}$ are scalars. In the same way, if $w L \subset \Pi$ then $E_{G}(\dot{w} D)$ has a basis $\left\{T_{v}^{\prime} \mid v \in W(w \delta)\right\}$ with

$$
T_{v}^{\prime}=\varepsilon_{w^{-1} v w}\left(p_{w^{-1} v w} \text { ind } v\right)^{1 / 2}\left(\dot{w} D^{0}\right)(\dot{v}) B_{\dot{w} D, \dot{v}} .
$$

(Note that $\dot{w} D^{0}$ is a projective representation of $\left\langle M_{w L}, W(w \delta)\right\rangle$ which extends $\dot{w} D$.)

(3.14.3) RemarK. By [9,(3.11)(iv)] and (3.14.1) above, $T_{v}$ does not depend on the coset representative $\dot{v}$. The same applies to $T_{v}^{\prime}$.

(3.15) Lemma. Let $w \in V_{\delta}$. The map $\tau_{w}: E_{G}(D) \rightarrow E_{G}(\dot{w} D)$ given by $\tau_{w}(T)=$ $B_{D, \dot{w}} T B_{D, \dot{w}}^{-1}$ is an isomorphism satisfying $\tau_{w}\left(T_{v}\right)=T_{w v w^{-1}}^{\prime}$ for all $v \in W(\delta)$.

Proof. It is trivial that $\tau_{w}$ is an isomorphism. If $v \in W(\delta)$

$$
\begin{aligned}
B_{D, \dot{w}} T_{v} & =\varepsilon_{v}\left(p_{v} \text { ind } v\right)^{1 / 2} B_{D, \dot{w}} D^{0}(\dot{v}) B_{D, \dot{v}} \\
& =\varepsilon_{v}\left(p_{v} \text { ind } v\right)^{1 / 2} D^{0}(\dot{v}) B_{\dot{v} D, \dot{w}} B_{D, \dot{v}} \quad([9,(3.15)]) \\
& =\varepsilon_{v}\left[p_{v}(\text { ind } w)^{-1}(\text { ind } w v)\right]^{1 / 2} D^{0}(\dot{v}) B_{D, \dot{w} v}
\end{aligned}
$$

by Lemma (3.14), since $N(w) \cap v \Gamma=N(w) \cap \Gamma=\varnothing$. However, in view of [9, (3.11)(iv)], Lemma (3.14) also gives

$$
B_{\dot{w} D, \dot{w} \dot{w} \dot{w}^{-1}} B_{D, \dot{w}}=\left[\left(\text { ind } w v w^{-1}\right)^{-1}(\text { ind } w)^{-1}(\text { ind } w v)\right]^{1 / 2} B_{D, \dot{w} \dot{v}},
$$

since $N\left(w^{-1}\right) \cap w \Gamma=\varnothing$. Thus

$$
\tau_{w}\left(T_{v}\right)=\varepsilon_{v}\left(p_{v} \text { ind } w v w^{-1}\right)^{1 / 2} D^{0}(\dot{v}) B_{\dot{w} D, \dot{w} \dot{v} \dot{w}^{-1}}
$$

But

$$
\begin{aligned}
D^{0}(\dot{v}) B_{\dot{w} D, \dot{w} \dot{v} \dot{w}^{-1}} & =\left(\dot{w} D^{0}\right)\left(\dot{w} \dot{v} \dot{w}^{-1}\right) B_{\dot{w} D, \dot{w} \dot{v} \dot{w}^{-1}} \\
& =\left(\dot{w} D^{0}\right)\left(\dot{w} \dot{v} \dot{w}^{-1} h\right) B_{\dot{w} D, \dot{w} \dot{w} \dot{w}^{-1}} h
\end{aligned}
$$


for any $h \in B \cap N$, by [9,(3.11)(iv)]. In partricular $h$ may be chosen so that $\dot{w} \dot{v} \dot{w}^{-1} h=\dot{u}, u=w v w^{-1}$, and then (3) above gives $\tau_{w}\left(T_{v}\right)=T_{u_{v} w^{-1}}^{\prime}$.

(3.16) THEOREM. Let $w \in V_{\delta}, w L \subset J \subset \Pi$. The image of $E_{J}(\dot{w} D) \quad(=$ $\left.\operatorname{End}_{P_{J}}\left(\operatorname{Ind}\left(P_{w L} \rightarrow P_{J} ; \overparen{\dot{w} D}\right)\right)\right)$ under the injection $E_{J}(\dot{w} D) \rightarrow E_{G}(\dot{w} D)$ of $(1.11)$ is the $\mathrm{C}$-linear span of $\left\{T_{v}^{\prime} \mid v \in W_{J} \cap W(w \delta)\right\}$. Furthermore the $\mathbf{C}$-linear span of $\left\{T_{v} \mid v \in\right.$ $\left.W_{J}^{n} \cap W(\delta)\right\}$ is a subalgebra of $E_{G}(D)$ which is mapped onto the image of $E_{J}(\dot{w} D)$ by the isomorphism $\tau_{\mathfrak{u}}$ of (3.15).

Proof. We have

$$
\widetilde{\mathscr{q}}_{G}(\dot{w} D)=\left\{f: P_{J} \rightarrow V \mid f(x y)=(\widetilde{\dot{w} D})(x) f(y) \text { for all } x \in P_{w \cdot L}, y \in P_{J}\right\} .
$$

Define similarly

$$
\widetilde{\mathscr{F}}_{J}(\dot{w} D)=\left\{f: P_{J} \rightarrow V \mid f(x y)=(\widetilde{\dot{w} D})(x) f(y) \text { for all } x \in P_{w L}, y \in P_{J}\right\} .
$$

By (1.10) there is a natural $\mathbf{C} P_{J}$-homomorphism $\pi: \mathscr{F}_{G}(\dot{w} D) \rightarrow \mathscr{F}_{J}(\dot{w} D)$ which takes each $f \in F_{G}(\dot{w} D)$ to its restriction to $P_{J}$. The image of $E_{J}(\dot{w} D)$ under the injection $E_{J}(\dot{w} D) \rightarrow E_{G}(\dot{w} D)$ is (by (1.11)) $\left\{T \in E_{G}(\dot{w} D) \mid T(\operatorname{ker} \pi) \subset \operatorname{ker} \pi\right\}$ (where $\pi$ is the surjection above). But $\operatorname{dim} E_{J}(\dot{w} D)=\left|W_{J} \cap W(w \delta)\right|$; so to show that $\left\{T_{v}^{\prime} \mid v \in W_{J}\right.$ $\cap W(w \delta)\}$ is a basis for this space it suffices to show that $T_{v}^{\prime}(\operatorname{ker} \pi) \subset \operatorname{ker} \pi$ for all $v \in W_{J} \cap W(w \delta)$.

Let $f \in \operatorname{ker} \pi$ and $x \in P_{J}$. Then for all $y \in U_{v w L}$ we have $\dot{v}^{-1} y \in P_{J}$ (since $w L\llcorner J$ and $\left.v \in W_{J}\right)$, and $\dot{v}^{-1} y x \in P_{J}$. Since the restriction of $f$ to $P_{J}$ is zero $f\left(\dot{v}^{-1} y x\right)=0$ and by the definition of $B_{\dot{w} D, \dot{v}}$ we have $\left(B_{\dot{w} D, \dot{v}} f\right)(x)=0$. But

$$
\left(T_{v}^{\prime} f\right)(x)=c\left[\left(\dot{w} D^{0}\right)(\dot{v})\right]\left[\left(B_{\dot{w} D, \dot{v}} f\right)(x)\right]
$$

for some scalar $c$, and we deduce that $T_{v}^{\prime} f$ restricted to $P_{J}$ is zero, as required.

The rest is trivial.

Henceforth we shall regard $E_{J}(\dot{w} D)$ as a subalgebra of $E_{G}(\dot{w} D)$ in accordance with (3.16).

4. Generic algebras and their representations. We maintain the notation of $\S 3$. Let $\left\{u_{a} \mid a \in \Delta\right\}$ be a set of indeterminates, with $u_{a}=u_{b}$ if and only if $a=w(b)$ for some $w \in W(\delta)$. Let $\mathcal{K}$ be the algebraic closure of the quotient field of the polynomial ring $\mathbf{C}\left[u_{a} \mid a \in \Delta\right]$. Let $\mathscr{R}$ be the multiplicative subgroup of $\mathscr{K}$ generated by $\left\{u_{a} \mid a \in \Delta\right\}$ and $\mathscr{D}$ the integral closure in $\mathscr{K}$ of the subring $\mathscr{D}_{0}=\{f / m \mid m \in \mathscr{N}$, $\left.f \in \mathbf{C}\left[u_{a} \mid a \in \Delta\right]\right\}$. ( $\mathscr{D}_{0}$ is the localization of the polynomial ring at $\mathscr{N}$.) Let $A$ be a free $\left(\mathcal{D}\right.$-module with basis $\left\{a_{x} \mid x \in W(\delta)\right\}$. For $w \in W(\delta)$, let $n(w)$ be the number of $a \in \Gamma^{+}$with $w(a) \notin \Gamma^{+}$(that is, $\left.n(w)=|N(w) \cap \Gamma|\right)$.

(4.1) THEOREM. There exists a unique $\mathbb{D}$-bilinear associative multiplication on $A$ such that for all $x \in C(\delta), w \in W(\delta), a \in \Delta$,

$$
\begin{gathered}
a_{x} a_{w}=\mu(x, w) a_{x w}, \\
a_{w} a_{x}=\mu(w, x) a_{w x}, \\
a_{v} a_{w}= \begin{cases}a_{v w} & \text { if } w^{-1} a \in \Gamma^{+}, \\
u_{a} a_{v w}+\left(u_{a}-1\right) a_{w} & \text { if } w^{-1} a \notin \Gamma^{+},\end{cases}
\end{gathered}
$$




$$
a_{w} a_{v}= \begin{cases}a_{w v} & \text { if } w a \in \Gamma^{+}, \\ u_{a} a_{w v}+\left(u_{a}-1\right) a_{w} & \text { if } w a \notin \Gamma^{+},\end{cases}
$$

where $v=v(a, L)$ and $\mu$ is as in Theorem (3.10).

Proof. We employ a method due to Tits (see [10]) and only sketch the proof.

For each $a \in \Delta$ define $\lambda_{a}$ and $p_{a} \in \operatorname{End}_{\mathscr{D}}(A)$ by

$$
\begin{aligned}
& \lambda_{a}\left(a_{w}\right)= \begin{cases}a_{v w} & \text { if } w^{-1} a \in \Gamma^{+}, \\
u_{a} a_{v w}+\left(u_{a}-1\right) a_{w} & \text { if } w^{-1} a \notin \Gamma^{+},\end{cases} \\
& \rho_{a}\left(a_{w}\right)= \begin{cases}a_{w v} & \text { if } w a \in \Gamma^{+}, \\
u_{a}\left(a_{w v}\right)+\left(u_{a}-1\right) a_{w} & \text { if } w a \notin \Gamma^{+},\end{cases}
\end{aligned}
$$

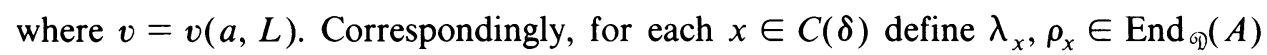
by

$$
\lambda_{x}\left(a_{w}\right)=\mu(x, w) a_{x w}, \quad \rho_{x}\left(a_{w}\right)=\mu(w, x) a_{w x} .
$$

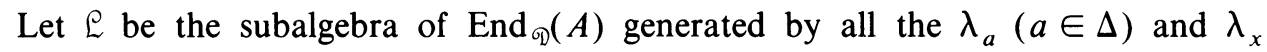
$(x \in C(\delta))$, and $\Re$ the subalgebra of $\operatorname{End}_{\mathscr{D}}(A)$ generated by all the $\rho_{a}$ and $\rho_{x}$.

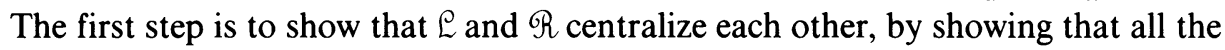
generators of $\mathcal{L}$ commute with all the generators of $R$. This is done by consideration of several cases, of which we present only two:

(1) Suppose $x \in C(\delta), a \in \Delta, w \in W(\delta)$ and $w a \notin \Gamma^{+}$. Then

$$
\begin{aligned}
\lambda_{x} \rho_{a}\left(a_{w}\right) & =\lambda_{x}\left(u_{a} a_{w v}+\left(u_{a}-1\right) a_{w}\right) \\
& =u_{a} \lambda_{x}\left(a_{w v}\right)+\left(u_{a}-1\right) \lambda_{x}\left(a_{w}\right) \\
& =\mu(x, w v) u_{a} a_{x w v}+\mu(x, w)\left(u_{a}-1\right) a_{x w} .
\end{aligned}
$$

But we also have $(x w)(a) \notin \Gamma^{+}$; so

$$
\rho_{a} \lambda_{x}\left(a_{w}\right)=\mu(x, w) \rho_{a}\left(a_{x w}\right)=\mu(x, w)\left(u_{a} a_{x w v}+\left(u_{a}-1\right) a_{x w}\right) .
$$

By Theorem (3.10), $\mu(x, w v)=\mu(x, w)$, and hence $\lambda_{x} \rho_{a}\left(a_{w}\right)=\rho_{a} \lambda_{x}\left(a_{w}\right)$.

(2) Suppose $a, b \in \Delta, w \in W(\delta), n(w v)=n(w)+1$, and $n(u w v)=n(u w)-1$, where $v=v(a, L)$ and $u=v(b, L)$. We have that $w a \in \Gamma^{+}$but $(u w)(a) \notin \Gamma^{+}$. Since $b \in \Delta$ it follows that $w a=b$. Hence $w v=u w, u_{a}=u_{b}$. Now

$$
\begin{aligned}
\lambda_{b} \rho_{a}\left(a_{w}\right) & =\lambda_{b}\left(a_{w v}\right)=u_{b} a_{u w v}+\left(u_{b}-1\right) a_{w v} \\
& =u_{a} a_{u w v}+\left(u_{a}-1\right) a_{w v}=\rho_{a}\left(a_{u w}\right)=\rho_{a} \lambda_{b}\left(a_{w}\right) .
\end{aligned}
$$

The next step is to observe that the $\mathscr{D}$-module maps $\mathcal{L} \rightarrow A ; \Re \rightarrow A$ given by $\lambda \mapsto \lambda\left(a_{1}\right), \rho \mapsto \rho\left(a_{1}\right)$ are bijective. Indeed, if $w=x v_{1} v_{2} \cdots v_{l}$ with $x \in C(\delta)$, $v_{i}=v\left(a_{i}, L\right), a_{i} \in \Delta$ and $n(w)=l$, then it is easily seen that

$$
\left(\lambda_{x} \lambda_{a_{1}} \cdots \lambda_{a_{l}}\right)\left(a_{1}\right)=a_{w}=\left(\rho_{a} \rho_{a_{l-1}} \cdots \rho_{a_{1}} \rho_{x}\right)\left(a_{1}\right) \text {, }
$$

so that the maps are surjective. Now if $\lambda \in L$ is such that $\lambda\left(a_{1}\right)=0$, then for all $\rho \in R$

$$
\lambda\left(\rho\left(a_{1}\right)\right)=\rho\left(\lambda\left(a_{1}\right)\right)=0
$$


and since $\rho \mapsto \rho\left(a_{1}\right)$ is surjective it follows that $\lambda=0$. This proves the injectivity of $\lambda \mapsto \lambda\left(a_{1}\right)$; a similar argument applies to the map $\rho \mapsto \rho\left(a_{1}\right)$.

We now define multiplication on $A$ by setting $\rho\left(a_{1}\right) \sigma\left(a_{1}\right)$ equal to $(\rho \sigma)\left(a_{1}\right)$ for all $\rho, \sigma \in R$ (so that $\rho \mapsto \rho\left(a_{1}\right)$ becomes an algebra isomorphism). It is easily checked that this multiplication has the required properties, and uniqueness is clear too.

If $f: \mathscr{D} \rightarrow \mathbf{C}$ is a homomorphism we make $\mathbf{C}$ into a right $\mathscr{Q}$-module by defining

$$
c \cdot r=c f(r), \quad r \in \mathscr{D}, c \in \mathbf{C} .
$$

Then the specialized algebra $A^{f}=\mathbf{C} \otimes_{\mathscr{D}} A$ is a $\mathbf{C}$-algebra with basis $\left\{a_{w f} \mid w \in W(\delta)\right\}$ where $a_{w f}=1 \otimes a_{w}$, and the structure constants are obtained by applying $f$ to the structure constants of $A$. That is, if $a_{w} a_{v}=\sum_{u} r_{w v u} a_{u}\left(r_{w v u} \in \mathcal{D}\right)$ then $a_{w f} a_{v f}=$ $\sum_{u} f\left(r_{w v u}\right) a_{u f}$.

(4.2) Lemma. Let $f_{0}, g_{0}: \mathscr{D}_{0} \rightarrow \mathbf{C}$ be the homomorphisms defined by $f_{0}\left(u_{a}\right)=p_{a}$, $g_{0}\left(u_{a}\right)=1(a \in \Delta)$. Then

(i) $f_{0}$ and $g_{0}$ have extensions $f$, $g$ to homomorphisms $\mathscr{D} \rightarrow \mathbf{C}$,

(ii) $\bar{f}: a_{w f} \mapsto T_{w}$ defines an isomorphism $A^{f} \rightarrow E_{G}(D)$,

(iii) $A^{g}$ is isomorphic to $(\mathrm{C} W(\delta))_{\mu}$.

Proof. (i) The proof of this may be found in Bourbaki $[2$, Chapter $5, \S 2.1]$. See also [5, Proposition (5.6)].

(ii) This is immediate on comparing the statement of Theorem (3.10) with the multiplication table of $A$.

(iii) We have $a_{w g} a_{v g}=\mu(w, v) a_{w v g}$ for any pair $w, v \in W(\delta)$. The result follows.

(4.3) Proposition. Let $w \in V_{\delta}$ (see 3.11) and suppose $w L \subset J \subset \Pi$. Let $A_{J, w}$ be the OD-linear span of $\left\{a_{v} \mid v \in W_{J}^{w} \cap W(\delta)\right\}$. Then

(i) $A_{J, w}$ is a subalgebra of $A$ and has a presentation analogous to that of (4.1), with $W(\delta), C(\delta), \Delta$ replaced by $W_{J}^{w} \cap W(\delta), W_{J}^{w} \cap C(\delta), \Lambda=\{a \in \Delta \mid w a \in\langle J\rangle\}$ respectively.

(ii) If $\tau_{w}$ is the isomorphism $E_{G}(D) \rightarrow E_{G}(\dot{w} D)$ defined in (3.15) then the image of $\bar{f}\left(A_{J, w}^{f}\right)\left(\subset E_{G}(D)\right)$ under $\tau_{w}$ is $E_{J}(\dot{w} D)=\operatorname{End}_{P_{J}}\left(\operatorname{Ind}\left(P_{w L} \rightarrow P_{J} ; \dot{w} D\right)\right)$.

Proof. (i) Let $A_{1}$ be the subalgebra of $A$ generated by $\left\{a_{x}, a_{v} \mid x \in W_{J}^{w} \cap C(\delta)\right.$, $v=v(a, L), a \in \Lambda\}$. We use induction on $n(u)$ to show that $a_{u} \in A_{1}$ for all $u \in W_{J}^{w} \cap W(\delta)$. If $n(u)=0$ then by (3.9), $u \in C(\delta)$ and the result is trivial. If $n(u)>0$ then by (3.13) we may write $u=t v$ with $t \in W_{J}^{w} \cap W(\delta), n(t)=n(u)-1$, and $v=v(a, L), a \in \Lambda$. The inductive hypothesis gives $a_{t} \in A_{1}$ and (4.1)(iv) gives $a_{u}=a_{t} a_{v}$; we deduce (since $a_{v} \in A_{1}$ ) that $a_{u} \in A_{1}$, as required. Thus $A_{J, w} \subset A_{1}$.

Now let $x \in W_{J}^{w} \cap C(\delta), a \in \Lambda, v=v(a, L), u \in W_{J}^{w} \cap W(\delta)$. Since $x \in C(\delta)$ and $a \in \Delta$ we may use (4.1) to obtain expressions for $a_{x} a_{u}, a_{u} a_{x}, a_{v} a_{u}$ and $a_{u} a_{v}$, and in particular we see that all these products lie in $A_{J, w}$ since $x u, u x, v u, u v$ and $u$ all lie in $W_{J}^{w} \cap W(\delta)$. Thus it follows that $A_{J, w}=A_{1}$, and moreover the expressions obtained for $a_{x} a_{u}, a_{u} a_{x}, a_{v} a_{u}$ and $a_{u} a_{v}$ show that $A_{J, w}$ has the presentation described in the statement above. 
(ii) This is a straightforward consequence of Theorem (3.16) and (i) above.

For $a \in \Gamma$ define $u_{a}=u_{w a}$ for any $w \in W(\delta)$ such that $w a \in \Delta$ (this is clearly independent of $w)$. For each $w \in W(\delta)$ define $u_{w}$ to be the product $\Pi u_{a}$ over $a \in N(w) \cap \Gamma$. Write $p_{w}=f\left(u_{w}\right)$, as in $[9,(4.9)]$.

For $a, b \in A$ define $(a, b) \in \mathcal{Q})$ to be the coefficient of $a_{1}$ when $a b$ is expressed as a linear combination of the $a_{w}, w \in W(\delta)$. Clearly $($,$) is \mathscr{D}$-bilinear, and associative in the sense that $(a b, c)=(a, b c)$.

(4.4) Lemma. (i) If $w, v \in W(\delta)$ then

$$
\left(a_{w}, a_{v}\right)= \begin{cases}0 & \text { if } v \neq w^{-1} \\ \mu(w, v) u_{w} & \text { if } v=w^{-1} .\end{cases}
$$

(ii) The form (, ) is symmetric and nondegenerate, and $\left\{a_{w} \mid w \in W(\delta)\right\}$ and $\left\{\mu\left(w, w^{-1}\right)^{-1} u_{w}^{-1} a_{w^{-1}} \mid w \in W(\delta)\right\}$ are dual bases with respect to it.

Proof. The second part is a trivial consequence of the first provided $\mu\left(w, w^{-1}\right)=$ $\mu\left(w^{-1}, w\right)$. For this we have

$$
\begin{aligned}
\mu\left(w, w^{-1}\right) & =\mu\left(w, w^{-1}\right) \mu(1, w) \quad(\operatorname{see}(3.10)) \\
& =\mu(w, 1) \mu\left(w^{-1}, w\right)=\mu\left(w^{-1}, w\right),
\end{aligned}
$$

as required. We are thus reduced to proving (i).

Use induction on $n(w)$. If $n(w)=0$ then $w \in C(\delta)$ (by (3.9)) and $a_{w} a_{v}=$ $\mu(w, v) a_{w v}$ (by (4.1)). Thus the coefficient of $a_{1}$ is 0 unless $w v=1$, in which case it is $\mu(w, v)=\mu(w, v) u_{w}$. So (i) holds in this case.

If $n(w)>0$ we may write $w=t u$ with $n(t)=n(w)-1$ and $u=v(a, L), a \in \Delta$. Then $\left(a_{w}, a_{v}\right)=\left(a_{t} a_{u}, a_{v}\right)=\left(a_{t}, a_{u} a_{v}\right)$, and since by (4.1)(iii) $a_{u} a_{v}$ is a $\mathcal{D}$-linear combination of $a_{v}$ and $a_{u v}$ it follows from the inductive hypothesis that $\left(a_{w}, a_{v}\right)=0$ unless $u v=t^{-1}$ or $v=t^{-1}$; that is, unless $w=v^{-1}$ or $v=t^{-1}$. If $v=t^{-1}$ then $v^{-1} a \in \Gamma^{+}($since $n(t u)=n(t)+1)$, and (4.1)(iii) gives $a_{u} a_{v}=a_{u v}$. So $\left(a_{w}, a_{v}\right)=$ $\left(a_{t}, a_{u v}\right)=0$ (since $\left.u v \neq t^{-1}\right)$ in this case too. Finally, suppose $w=v^{-1}$. Then $v^{-1} a \in \Gamma^{-}($since $n(w u)=n(t)=n(w)-1)$ and, using (4.1)(iii), we have

$$
\begin{aligned}
\left(a_{w}, a_{v}\right) & =\left(a_{t}, u_{a} a_{u v}+\left(u_{a}-1\right) a_{v}\right) \\
& =\mu\left(t, t^{-1}\right) u_{a} u_{t} \text { by the inductive hypothesis. }
\end{aligned}
$$

But $N(w) \cap \Gamma=\{a\} \cup u(N(t) \cap \Gamma)$, and it follows that $u_{w}=u_{a} u_{t}$. Since $\mu\left(w, w^{-1}\right)$ $=\mu\left(t u, u^{-1} t^{-1}\right)=\mu\left(t, t^{-1}\right)$ (by 3.10), this gives the required formula.

(4.5) LEMMA. The element $c=\Sigma \mu\left(w, w^{-1}\right)^{-1} u_{w}^{-1} a_{w} a_{w^{-1}}$ (sum over $w \in W(\delta)$ ) is invertible in $A \otimes_{\mathcal{D}} \mathcal{K}$.

Proof. The regular representation of $A \otimes \mathcal{K}$ yields an isomorphism $\gamma$ from $A \otimes \mathscr{K}$ to an algebra $\mathcal{Q}$ of $|W(\delta)| \times|W(\delta)|$ matrices over $\mathscr{K}$ in which the $a_{w}(w \in W(\delta))$ and $c$ are represented by matrices with entries in $\mathscr{D}_{0}$ with respect to the basis $\left\{a_{w} \mid w \in W(\delta)\right\}$. Applying the homomorphism $g_{0}$ yields the matrices which represent the $a_{w g}$ and $c_{g}=\Sigma \mu\left(w, w^{-1}\right)^{-1} u_{w}^{-1} a_{w g} a_{w^{-1} g}$ in the regular representation of $A^{g}$. But $c_{g}=|W(\delta)| a_{1 g}$ is represented by $|W(\delta)| I$ ( $I=$ identity matrix) which has nonzero determinant, and since this determinant is obtained by applying $g$ to the 
determinant of the matrix representing $c$ it follows that $c$ is represented by an invertible matrix. By the Cayley-Hamilton Theorem the inverse of $\gamma(c)$ lies in $\mathscr{Q}$ (it is a polynomial in $\gamma(c)$ ), and its inverse image under the isomorphism is the required inverse of $c$ in $A$.

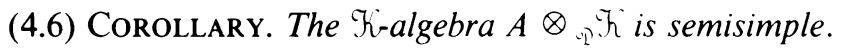

Proof. This follows from [6,71.6 and 71.1].

The next proposition provides the link between representations of $E_{G}(D)$ and projective representations of $W(\delta)$, by relating the representation theory of $A$ to that of the specialized algebras $A^{f}$ and $A^{g}$.

(4.7) Proposition. Let $h_{0}:$ OD $_{0} \rightarrow \mathbf{C}$ be a homomorphism and let $h:(1) \rightarrow \mathbf{C}$ be a fixed extension of $h_{0}$. Assume that the specialization $A^{h}$ is semisimple.

(i) For each irreducible $\mathfrak{K}$-character $\chi$ of $A \otimes_{0 \mathrm{D}} \mathcal{K}$ we have $\chi\left(a_{w}\right) \in \cup_{1}$ for all $w \in W(\delta)$.

(ii) If $\chi^{h}$ is defined by $\chi^{h}\left(a_{w h}\right)=h\left(\chi\left(a_{w}\right)\right)(w \in W(\delta))$ then $\chi^{h}$ is an irreducible character of $A^{h}$.

(iii) The map $\chi \mapsto \chi^{h}$ is a bijection between the set of irreducible Tr-characters of $A \otimes{ }_{\mathbb{N}} K$ and the set of irreducible $\mathbf{C}$-characters of $A^{h}$.

The proof may be found in [5, proof of (7.1)].

A major application of (4.7) is

(4.8) THEOREM. Suppose we are given the following data:

(a) $A$ subset $L \subset \Pi$, an irreducible cuspidal representation $D$ of $M_{L}$, with character $\delta$, and an extension $D^{0}$ of $D$ to $M^{0}=\left\langle M_{L}, W(\delta)\right\rangle$ (as in (3.5)) for which the associated 2-cocycle $\mu$ of $W(\delta)$ satisfies the equations in (3.10).

(b) Fixed extensions $f$ and $g: \mathscr{Q} \rightarrow \mathbf{C}$ of the homomorphisms $f_{0}$ and $g_{0}: \mathscr{D}_{0} \rightarrow \mathbf{C}$ defined in (4.2).

Then there exists a canonical bijection $\phi \mapsto \zeta_{\phi}$ from $\operatorname{Irr}_{\mu}(W(\delta))$ to the set of irreducible constituents of $\operatorname{Ind}\left(P_{L} \rightarrow G ; \tilde{\delta}\right)$.

Proof. From (4.2)(ii) and (iii) it follows that the specializations $A^{f}$ and $A^{g}$ are respectively isomorphic (canonically) to the complex algebras $E_{G}(D)=$ $\operatorname{End}_{G}\left(\operatorname{Ind}\left(P_{L} \rightarrow G ; D\right)\right)$ and $(\mathbf{C} W(\delta))_{\mu}$, which are both semisimple. Thus as a consequence of (4.7) we deduce the existence of a canonical bijection between the sets of irreducible characters of $E_{G}(D)$ and of $(\mathrm{CW}(\delta))_{\mu}$. However, by (1.14) the irreducible characters of $E_{G}(D)$ are in canonical bijective correspondence with the irreducible constituents of $\operatorname{Ind}\left(P_{L} \rightarrow G ; \tilde{\delta}\right)$, and the result follows.

Since we shall need to make use of the definition of the correspondence $\phi \mapsto \zeta_{\phi}$ of (4.8) we also state

(4.9) THEOREM. If $\phi$ and $\zeta_{\phi}$ are in correspondence as in (4.8) there is a unique K-character $\chi$ of $A$ such that for all $w \in W(\delta), \chi^{f}\left(a_{w f}\right)=\zeta^{\prime}\left(T_{W}\right)$ and $\chi^{g}\left(a_{w g}\right)=\phi(w)$ where $\zeta^{\prime}$ is the character of $E_{G}(D)$ which corresponds via (1.14) to the character $\zeta_{\phi}$ of $G$.

The statement (4.9) was proved in the course of proving (4.8). 
5. Representations of $G$ and of $W$-the Comparison Theorem. It is apparent from the material above, and particularly from Theorem (4.8), that the representation theory of $G$ is intimately connected with that of $W$ and its subgroups $W(\delta)$, the transition being accomplished by means of the "generic algebras" of $\S 4$. In this section we prove some results concerning $W(\delta)$ and its representation theory and give a refinement (Theorem (5.6)) of the correspondence given in Theorem (4.8). This leads to the statement of a multiplicity formula called the "Comparison Theorem" which we prove in the next section.

Notation is fixed as in the statement of (4.8).

(5.1) Definition. For $J \subset \Pi$ write $C_{L}(J)=\{w \in W \mid w L \subset\langle J\rangle\}$ (where, as in $\S 3,\langle J\rangle$ is the linear span of $J$ in the ambient Euclidean space $E$ ).

Note that Definition (5.1) is not consistent with the notation of [8], where $C_{I}(J)$ consisted of the set of inverses of the elements in our present $C_{L}(J)$. It is clear that $C_{L}(J)$ is a union of $\left(N_{W}\left(W_{J}\right)\right),\left(N_{W}\left(W_{L}\right)\right)$ double cosets, and hence a union of $\left(W_{J}, W(\delta)\right)$ double cosets. Note also that if $w \in W$, the character

$$
\left(\operatorname{Ind}\left(W_{J}^{w} \cap W(\delta) \rightarrow W(\delta) ; 1\right)\right)
$$

depends only on the $\left(W_{J}, W(\delta)\right)$ double coset to which $w$ belongs.

(5.2) Definition. The linear character $\alpha_{I}$ of $N_{W}\left(W_{L}\right)$ is defined (as in [8]) by $\alpha_{L}(w)=\operatorname{det} \bar{w}$, where $\bar{n}$ is the restriction of $w$ to $\langle L\rangle^{\perp}$, the orthogonal complement of $\langle L\rangle$ in $E$.

The character $\alpha_{l}$. may be thought of as a "sign character" for $N_{W}\left(W_{L}\right) / W_{L}$. We use " $\alpha_{I}$ " also to denote its restriction to any subgroup of $N_{W}\left(W_{L}\right)$.

From Theorem A of [8] we have

(5.3) Theовем. For each $J \subset \Pi$ let $S_{\delta}(J)$ be any fixed set of representatives of the $\left(W_{J}, W(\delta)\right)$ double cosets contained in $C_{L}(J)$. Then we have

$$
\sum_{J(11}(-1)^{|,|} \sum_{w} \operatorname{Ind}\left(W_{J}^{w \prime} \cap W(\delta) \rightarrow W(\delta) ; 1\right)=(-1)^{|L|} \alpha_{L},
$$

where the inner sum is over $w \in S_{\delta}(J)$.

This theorem will be applied later to the study of "duality" for characters of $G$.

(5.4) Lemma. Each $\left(W_{J}, W(\delta)\right)$ double coset in $C_{L}(J)$ contains an element $w$ with $w L \subset J$.

Proof. Any such double coset clearly contains an element $w$ such that $w$ is the shortest element in $W_{J} w$. For such a $w$ the (linear) map $a \mapsto w^{-1} a$ from $\langle J\rangle \cap \Sigma$ to $\Sigma$ is order preserving (it takes positive roots to positive roots). Thus if $a$ is not a fundamental root then neither is $w^{-1} a$, whence $w L$ consists of fundamental roots and is therefore contained in $J$.

(5.5) Corollary. Each $\left(W_{J}, W(\delta)\right)$ double coset in $C_{L}(J)$ contains an element $w$ satisfying $w L \subset J$ and $w \in V_{\delta}$ (that is, $w \Gamma^{+} \subset \Sigma^{+}-$see (3.11)).

Proof. This follows immediately from (5.4) and (3.12). 
(5.6) ThEOREM. Assume we are given the data (a) and (b) of Theorem (4.8). If $J \subset \Pi$ and $w \in V_{\delta}$ with $w L \subset J$ then there is a canonical bijection $\psi \mapsto \eta_{\psi}$ from $\operatorname{Irr}_{w \mu}\left(W_{J} \cap{ }^{w} W(\delta)\right)$ to $S$, the set of irreducible constituents of $\operatorname{Ind}\left(P_{w L} \cap M_{J} \rightarrow M_{J} ; w \delta\right)$.

Proof. Applying (4.3)(i) and (4.7) with $A_{J, w}$ in place of $A$, each character $\psi \in \operatorname{Irr}_{w \mu}\left(W_{J} \cap{ }^{w} W(\delta)\right)$ canonically determines an irreducible $\mathcal{F}_{\mathcal{K}}$-character $\chi$ of $A_{J,{ }^{*}}$ $\otimes \mathcal{K}$ such that

$$
\psi\left(w u w^{-1}\right)=g\left(\chi\left(a_{u}\right)\right) \quad\left(u \in W_{J}^{w^{\prime}} \cap W(\delta)\right) .
$$

Similarly, using (4.3)(ii) and (4.7), $\chi$ canonically determines an irreducible character $\chi^{\prime}$ of $E_{J}(\dot{w} D)=\operatorname{End}_{P_{J}}\left(\operatorname{Ind}\left(P_{w \cdot L} \rightarrow P_{J} ; \overparen{\dot{w} D}\right)\right.$ with

$$
\chi^{\prime}\left(\tau_{w} T_{u}\right)=f\left(\chi\left(a_{u}\right)\right) \quad\left(u \in W_{J}^{w} \cap W(\delta)\right) .
$$

By (1.14), $\chi^{\prime}$ corresponds canonically to an irreducible constituent $\eta$ of

$$
\operatorname{Ind}\left(P_{w \cdot L} \rightarrow P_{J} ; w \delta\right) \text {. }
$$

Define $\eta_{\psi}$ to be the restriction of $\eta$ to $M_{J}$. It is clear that the map $\psi \mapsto \eta_{\psi}$ has the required properties.

Theorem (5.6) may also be proved by applying Theorem (4.8) with $C$ replaced by $M_{J}, P_{L}$ by $P_{w L} \cap M_{J}$ (which is a standard parabolic subgroup of $M_{J}$ with standard Levi component $M_{u^{\prime} \cdot L} \subset M_{J}$ ) and $\delta$ by $w \delta$. One checks that the data (a) and (b) of (4.8) naturally determine corresponding data in this new situation.

Note that if $w L \subset J \subset \Pi$ and $v \in w W(\delta)$ then $W_{J} \cap{ }^{w} W(\delta)=W_{J} \cap^{v} W(\delta)$ and (by the results of $\S 2$ ) the cocycles $w \mu$ and $v \mu$ are equivalent. Moreover, by (2.2) there is a natural bijection $\rho: \psi \mapsto \rho \psi$ from the irreducible $w \mu$-characters to the irreducible $v \mu$-characters defined by

$$
(\rho \psi)(u)=\mu\left(u^{w}, x\right)^{-1} \mu\left(x, u^{v}\right) \psi(u) \quad\left(u \in W_{J} \cap^{v} W(\delta)\right),
$$

where $x=w^{-1} v \in W(\delta)$. (In the notation of $\S 2$,

$$
\left.\bar{x}\left[v^{-1}(\rho \psi)\right]=w^{-1} \psi \in \operatorname{Irr}_{\mu}\left(W_{J}^{w} \cap W(\delta)\right) .\right)
$$

(5.7) Lemma. Let $w \in V_{\delta}$ with $w L \subset J \subset \Pi$ and suppose $v \in V_{\delta} \cap w W(\delta)$. Let $\psi \mapsto \eta_{\psi}$ be the bijection defined in Theorem (5.6) and let $\theta \mapsto \eta_{\theta}$ from $\operatorname{Irr}_{v \mu}\left(W_{J} \cap{ }^{v} W(\delta)\right)$ to $\operatorname{Irr}\left(M_{J}\right)$ be defined in the same way with $w$ replaced by $v$. Then $\eta_{\rho \psi}=\eta_{\psi}(\rho$ as in (5.6.1)) for all $\psi \in \operatorname{Irr}_{w \mu}\left(W_{J} \cap{ }^{w} W(\delta)\right)$.

Proof. Since $v \in w W(\delta)$ we have $v \Gamma=w \Gamma$, and since $v, w \in V_{\delta}$ we have $v \Gamma^{+} \subset$ $\Sigma^{+}, w \Gamma^{+} \subset \Sigma^{+}$. Hence $v \Gamma^{+}=w \Gamma^{+}$, and so $x=w^{-1} v$ lies in $C(\delta)$. For any $\mathcal{K}$-character $\chi$ of $A_{J, v} \otimes \mathcal{K}$ we can define a $K_{\text {-character }} \bar{x} \chi$ of $A_{J, w} \otimes \mathcal{K}$ by

$$
(\bar{x} \chi)\left(a_{u}\right)=\chi\left(a_{x}^{-1} a_{u} a_{x}\right)=\mu(u, x) \mu\left(x, u^{x}\right)^{-1} \chi\left(a_{x^{-1} u x}\right)
$$

for all $u \in W_{J}^{w} \cap W(\delta)$. From the proof of (5.6) $\bar{x} \chi$ corresponds to some $\psi \in$ $\operatorname{Irr}_{w \mu}\left(W_{J} \cap{ }^{w} W(\delta)\right)$, and we have for $u \in W_{J}^{v} \cap W(\delta)$,

$$
\begin{aligned}
(\rho \psi)\left(v u v^{-1}\right) & =\mu\left(x u x^{-1}, x\right)^{-1} \mu(x, u) \psi\left(v u v^{-1}\right) \\
& =\mu\left(x u x^{-1}, x\right)^{-1} \mu(x, u) g\left[(\bar{x} \chi)\left(a_{x u x^{-1}}\right)\right]=g\left(\chi\left(a_{u}\right)\right) .
\end{aligned}
$$


Thus $\rho \psi$ is the $v \mu$-character of $W_{J} \cap^{v} W(\delta)$ corresponding to the character $\chi$ of $A_{J, v} \otimes \mathfrak{K}$.

Similarly the characters $\chi, \bar{x} \chi$ yield characters $\chi^{\prime},(\bar{x} \chi)^{\prime}$ of $E_{J}(\dot{v} D), E_{J}(\dot{w} D)$ such that for $u \in W_{J}^{v} \cap W(\delta), \chi^{\prime}\left(\tau_{v} T_{u}\right)=f\left(\chi\left(a_{u}\right)\right)$, and for $u \in W_{J}^{u^{\prime}} \cap W(\delta)$,

$$
\begin{aligned}
(\bar{x} \chi)^{\prime}\left(\tau_{u} T_{u}\right) & =f\left((\bar{x} \chi)\left(a_{u}\right)\right)=\mu(u, x) \mu\left(x, u^{x}\right)^{-1} f\left(\chi\left(a_{x^{-1} u x}\right)\right) \\
& =\mu(u, x) \mu\left(x, u^{x}\right)^{-1} \chi^{\prime}\left(\tau_{v} T_{x^{-1} u x}\right) \\
& =\chi^{\prime}\left(\tau_{v}\left(T_{x}^{-1} T_{u} T_{x}\right)\right) .
\end{aligned}
$$

But by (3.14), (3.14.1) and [9,(3.15)(ii)] we have for some scalar $c$,

$$
B_{D, \dot{x}} T_{x}=c D^{0}(\dot{x}) B_{D, \dot{x} \cdot \dot{x}}=c D^{0}\left(\dot{w}^{-1} \dot{v}\right) B_{D, \dot{v}} \quad(\text { by }[9,(3.11)(\mathrm{iv})])
$$

and hence $\tau_{v}\left(T_{x}^{-1} T_{u} T_{x}\right)=D^{0}\left(\dot{w}^{-1} \dot{v}\right)^{-1}\left(\tau_{u} T_{u}\right) D^{0}\left(\dot{w}^{-1} \dot{v}\right)$. Thus we have $(\bar{x} \chi)^{\prime}(T)=$ $\chi^{\prime}\left(\sigma^{-1} T \sigma\right)$ for all $T \in E_{J}(\dot{w} D)$ where $\sigma: \widetilde{\mathscr{F}}_{J}(\dot{v} D) \rightarrow \mathscr{F}_{J}(\dot{w} D)$ is the $\mathbf{C} P_{J}$-isomorphism defined by $(\sigma f)(x)=D^{0}\left(\dot{w}^{-1} \dot{v}\right) f(x)$ (for $x \in P_{J}, f \in \widetilde{\mathcal{F}}_{J}(\dot{v} D)$ ). By $(1.15) \chi^{\prime}$ and $(\bar{x} \chi)^{\prime}$ correspond to the same character of $P_{J}$; that is, $\eta_{\rho \psi}=\eta_{\psi}$, as required.

Now suppose that $w$ is an arbitrary element of $W$ satisfying $w L \subset J \subset \Pi$. By (3.12) $w W(\delta) \cap V_{\delta} \neq \varnothing$. For any $v \in w W(\delta) \cap V_{\delta}$, there exist bijections $\psi \mapsto \rho \psi$ from $\operatorname{Irr}_{u^{\prime} \mu}\left(W_{J} \cap^{{ }^{\prime}} W(\delta)\right.$ ) to $\operatorname{Irr}_{v \mu}\left(W_{J} \cap{ }^{v} W(\delta)\right.$ ) (see (5.6.1)) and $\theta \mapsto \eta_{\theta}$ from $\operatorname{Irr}_{v \mu}\left(W_{J} \cap^{{ }^{\prime}} W(\delta)\right)$ to $S$ (see (5.6)). Define $\psi \mapsto \eta_{\psi}$ from $\operatorname{Irr}_{w \mu}\left(W_{J} \cap{ }^{w} W(\delta)\right)$ to $S$ to be the composite of these.

(5.8) Corollary. For any $w \in W$ such that $w L \subset J \subset \Pi$ the map $\psi \mapsto \eta_{w \psi}$ is a canonical bijection from $\operatorname{Irr}_{\mu}\left(W_{J}^{\mu^{*}} \cap W(\delta)\right)$ to $S$.

Proof. By the results of $\S 2, \psi \mapsto w \psi$ is a bijection from $\operatorname{Irr}_{\mu}\left(W_{J}^{w} \cap W(\delta)\right)$ to $\operatorname{Irr}_{{ }^{\prime \mu}}\left(W_{J} \cap^{{ }^{*}} W(\delta)\right)$, and so $\psi \mapsto \eta_{w^{*} \psi}$ is a bijection from $\operatorname{Irr}_{\mu}\left(W_{J}^{w} \cap W(\delta)\right)$ to $S$. We must show that this is independent of the choice of $v \in w W(\delta) \cap V_{\delta}$.

Let $v_{1}, v_{2} \in w W(\delta) \cap V_{\delta}$ and let $\rho_{1}, \rho_{2}$ be defined as in (5.6.1) with $v_{1}, v_{2}$ in place of $v$. Let $\psi \in \operatorname{Irr}_{\mu}\left(W_{J}^{\mu^{*}} \cap W(\delta)\right)$ and let $\psi_{i}=\rho_{i}(w \psi) \in \operatorname{Irr}_{v, \mu}\left(W_{J} \cap^{v_{i}} W(\delta)\right)$. We must show that $\eta_{\psi_{1}}=\eta_{\psi_{2}}$. A short calculation shows that $\psi=\bar{x}_{i}\left(v^{-1} \psi_{i}\right)(i=1,2)$, and by (2.3)(a), $v_{2}^{-1} \psi_{2}=\overline{x_{2}^{-1} x_{1}}\left(v_{1}^{-1} \psi_{1}\right)$. The conclusion now follows by applying (5.7) with $v_{1}, v_{2}$ replacing $v, w$ respectively and $\psi_{2}$ replacing $\psi$.

We come now to the statement of our main theorem (the Comparison Theorem).

(5.9) TheOREM. Suppose $L \subset \Pi$ (the set of fundamental roots), $\delta$ is an irreducible cuspidal character of $M_{L}$ and $\mu$ is the 2-cocycle of the ramification group $W(\delta)$ described in (3.10). Let $w \in W$ be such that $w L \subset J \subset \Pi$. Take $\phi \in \operatorname{Irr}_{\mu}(W(\delta))$, $\psi \in \operatorname{Irr}_{\mu}\left(W_{J}^{w} \cap W(\delta)\right)$ and let $\zeta_{\phi}, \eta_{w \psi}$ be the corresponding irreducible characters of $G$ and $M_{J}$ respectively (see (4.8) and (5.8)). Then

$$
\left.\left(\operatorname{Res}\left(G \rightarrow P_{J} ; \zeta_{\phi}\right), \eta_{w \psi}\right)=\operatorname{Res}\left(W(\delta) \rightarrow W_{J}^{w} \cap W(\delta) ; \phi\right), \psi\right) .
$$

Equivalently (by Frobenius reciprocity)

$$
\left(\zeta_{\phi}, \operatorname{Ind}\left(P_{J} \rightarrow G ; \tilde{\eta}_{w \psi}\right)\right)=\left(\phi, \operatorname{Ind}\left(W_{J}^{w} \cap W(\delta) \rightarrow W(\delta) ; \psi\right)\right)
$$

or, to use an alternative notation,

$$
\left(\zeta_{\phi}, \tilde{\eta}_{w \psi}^{G}\right)_{G}=\left(\phi, \psi^{W(\delta)}\right)_{W(\delta)}
$$


The result therefore compares a multiplicity in $G$ with a multiplicity in $W$; hence its name.

(5.10) Remarks. (i) The definition of $\eta_{w \psi}$ gives $\eta_{w \psi}=\eta_{v \bar{x} \psi}$ if $x \in W(\delta)$ is such that

$$
v=w x^{-1} \in V_{\delta} .
$$

It follows that $\eta_{(w y) \psi}=\eta_{w(\bar{y} \psi)}$ for all $y \in W(\delta)$. Now if (5.9.2) holds for some $w$ and all $\psi \in \operatorname{Irr}_{\mu}\left(W_{J}^{w} \cap W(\delta)\right)$ then we have for any $y \in W(\delta), \psi \in \operatorname{Irr}_{\mu}\left(W_{J}^{w y} \cap W(\delta)\right)$,

$$
\begin{aligned}
\left(\zeta_{\phi}, \operatorname{Ind}\left(P_{J} \rightarrow G ; \tilde{\eta}_{(x y) \psi}\right)\right) & =\left(\zeta_{\phi}, \operatorname{Ind}\left(P_{J} \rightarrow G ; \tilde{\eta}_{w(\bar{y} \psi)}\right)\right) \\
& =\left(\phi, \operatorname{Ind}\left(W_{J}^{w} \cap W(\delta) \rightarrow W(\delta) ; \bar{y} \psi\right)\right) \\
& =\left(\phi, \operatorname{Ind}\left(\left(W_{J}^{w} \cap W(\delta)\right)^{y} \rightarrow W(\delta) ; \psi\right)\right) \quad \text { by }(2.3)(\mathrm{b}) \\
& =\left(\phi, \operatorname{Ind}\left(W_{J}^{w y} \cap W(\delta) \rightarrow W(\delta) ; \psi\right)\right),
\end{aligned}
$$

so that (5.9.2) holds for wy. Hence it will suffice to prove Theorem (5.9) for any fixed choice of $w$ within a given $w W(\delta)$ coset.

(ii) $\eta \mapsto \tilde{\eta}$ is a bijection from the constituents of

$$
\operatorname{Ind}\left(P_{w L} \cap M_{J} \rightarrow M_{J} ; \widetilde{w \delta}\right)
$$

to the constituents of $\operatorname{Ind}\left(P_{w L} \rightarrow P_{J} ; \widetilde{w \delta}\right)$. Thus $\psi \mapsto \tilde{\eta}_{w \psi}$ is a bijection from

$$
\operatorname{Irr}_{\mu}\left(W_{J}^{w} \cap W(\delta)\right)
$$

to the constituents of $\operatorname{Ind}\left(P_{w L} \rightarrow P_{J} ; \widetilde{w \delta}\right)$.

(iii) The characters $\eta_{\phi}, \eta_{w \psi}$ depend upon the chosen extension of the specialization map $f_{0}: \mathscr{D}_{0} \rightarrow \mathbf{C}$ (which takes $u_{a}$ to $p_{a}$ ). However, Theorem (5.9) shows that the multiplicity $\left(\zeta_{\phi}, \operatorname{Ind}\left(W_{J}^{w} \cap W(\delta) \rightarrow W(\delta) ; w \psi\right)\right)$ is independent of this choice.

(iv) Two special cases of (5.9) have been dealt with by other authors: the case of principal series ( $L$ empty, $\delta=1$ ) was treated by Curtis in [3] while McGovern [12] proved (5.9) in the generalized principal series case ( $L$ empty, $\delta \neq 1$ ). The same remark applies to the duality theorem below (Theorem (7.5)).

6. Proof of the Comparison Theorem. In this section we give a proof of Theorem (5.9). We take as fixed the hypothesis of (5.9) and assume (without loss of generality, by $(5.10)(\mathrm{i})$ and (3.12)) that $w \in V_{\delta}$. Recall that $\operatorname{Ind}\left(P_{L} \rightarrow G ; \tilde{\delta}\right)=\operatorname{Ind}\left(P_{w L} \rightarrow G ; \widetilde{w \delta}\right)$ (see (3.3)).

Write $\zeta=\zeta_{\phi}$, the constituent of

$$
\operatorname{Ind}\left(P_{L} \rightarrow G ; \tilde{\delta}\right)
$$

which corresponds (as in (4.8)) to the character $\phi \in \operatorname{Irr}_{\mu}\left(W(\delta)\right.$ ), and let $\eta=\tilde{\eta}_{w \psi}$ be the constituent of

$$
\operatorname{Ind}\left(P_{w L} \rightarrow P_{J} ; w \delta\right)
$$

which corresponds to $\psi \in \operatorname{Irr}_{\mu}\left(W_{J}^{w} \cap W(\delta)\right.$ ) (see (5.10)(ii)). Let $\zeta^{\prime}$ and $\eta^{\prime}$ respectively be the corresponding irreducible characters of $E_{G}(D)$ and $E_{J}(\dot{w} D)$ (see (1.14)).

Define $E=\bar{f}\left(A_{J, w}^{f}\right)$; this is the linear span of $\left\{T_{v} \mid v \in W_{J}^{w} \cap W(\delta)\right\}$ (see (4.2)). By (4.3) $E$ is the subalgebra of $E_{G}(D)$ which is mapped onto $E_{J}(\dot{w} D)$ by the 
isomorphism $\tau_{w}: E_{G}(D) \rightarrow E_{G}(\dot{w} D)$. The following diagram summarises the situation:

$$
\begin{array}{ccccc}
A^{f} & \stackrel{\bar{f}}{\rightarrow} & E_{G}(D) & \stackrel{\tau_{w}}{\rightarrow} & E_{G}(\dot{w} D)=\operatorname{End}_{G}\left(\widetilde{w}_{P_{w}}^{G}\right) \\
\uparrow & & \uparrow & & \uparrow \\
A_{J . w}^{f} & \stackrel{\bar{f}}{\rightarrow} & E & \stackrel{\tau_{w}}{\rightarrow} & E_{J}(\dot{w} D)=\operatorname{End}_{P_{J}}\left(\widetilde{w}_{\dot{w} P_{P_{w}}^{P_{J}}}\right)
\end{array}
$$

\section{FIGURE 1}

In this commutative diagram, the horizontal arrows are isomorphisms and the vertical arrows are inclusions. The map $\iota$ is the natural inclusion of endomorphism algebras (see (1.11) and (4.3)(ii)).

Since $E_{J}(\dot{w} D)$ is not a subalgebra of $E_{G}(D)$, we may not speak of the "multiplicity of $\eta^{\prime}$ in the restriction of $\zeta^{\prime \prime \prime}$, but need to first pull $\eta^{\prime}$ back to $E$. The result is

(6.1) Proposition. The multiplicity of $\eta^{\prime} \tau_{w} \in \operatorname{Irr}(E)$ in the restriction of $\zeta^{\prime}$ equals the multiplicity of $\eta \in \operatorname{Irr}\left(P_{J}\right)$ in the restriction of $\zeta$.

Proof. Since $\tau_{w}(T)=\sigma T \sigma^{-1}\left(T \in E_{G}(D)\right)$ where $\sigma=B_{D, \dot{w}}$ is an isomorphism $\mathscr{F}_{G}(D) \rightarrow \mathscr{F}_{G}(\dot{w} D)$ it follows from (1.15) that the characters $\zeta^{\prime}$ of $E_{G}(D)$ and $\zeta^{\prime} \tau_{w}^{-1}$ of $E_{G}(\dot{w} D)$ correspond to the same character of $G$. By (1.14) applied with $H=P_{w L}$, $K=P_{J}, \chi=\widetilde{w \delta}, E_{G}=E_{G}(\dot{w} D)$ and $E_{K}=E_{J}(\dot{w} D)$, we deduce that the multiplicity of $\eta$ in the restriction of $\zeta$ equals the multiplicity of $\eta^{\prime}$ in the restriction $\zeta^{\prime} \tau_{w}^{-1}$. Composing with the isomorphism $\tau_{w}$, this equals the multiplicity of $\eta^{\prime} \tau_{w}$ in the restriction of $\zeta^{\prime}$.

(6.2) LEMMA. With notation as in $\$ 4$ ( $A$ the generic algebra, $f$ and $g$ specialization maps, etc.) let $\chi \in \operatorname{Irr}(A \otimes \mathscr{K})$ and $\xi \in \operatorname{Irr}\left(A_{J, w} \otimes \mathscr{K}\right)$. Then the multiplicity of $\xi^{f}$ $\left(\right.$ resp $\left.\xi^{g}\right)$ in the restriction of $\chi^{f}\left(\right.$ resp $\left.\chi^{g}\right)$ equals the multiplicity of $\xi$ in the restriction of $\chi$.

Proof. Write $\operatorname{Res}\left(A \otimes \mathcal{K} \rightarrow A_{J, w} \otimes \mathcal{K} ; \chi\right)=m_{1} \xi_{1}+m_{2} \xi_{2}+\cdots+m_{l} \xi_{\text {, where the }}$ $\xi_{i}$ form a complete set of irreducible $\mathcal{K}$-characters of the semisimple $\mathcal{K}$-algebra $A_{J, w} \otimes \mathcal{K}$ (that $A_{J, w} \otimes \mathscr{K}$ is semisimple follows from (4.3)(i) and (4.6)). Changing notation if necessary, assume $\xi_{1}=\xi$. We have that $\xi_{1}^{f}, \ldots, \xi_{l}^{f}$ form a complete set of distinct irreducible C-characters of $A_{J, w}^{f}$. Moreover

$$
\begin{aligned}
\operatorname{Res}\left(A^{f} \rightarrow A_{J, w}^{f} ; \chi^{f}\right) & =\left[\operatorname{Res}\left(A \otimes \mathscr{K} \rightarrow A_{J, w} \otimes \mathcal{K} ; \chi\right)\right]^{f} \\
& =m_{1} \xi_{1}^{f}+\cdots+m_{l} \xi_{l}^{f} .
\end{aligned}
$$

Thus the multiplicity of $\xi$ in the restriction of $\chi$ and the multiplicity of $\xi^{f}$ in the restriction of $\chi^{f}$ are both equal to $m_{1}$; similarly for $\xi^{g}$ and $\chi^{g}$.

Now by (4.9) there is a unique irreducible $\mathcal{K}$-character $\chi$ of $A$ such that $\zeta^{\prime}\left(T_{v}\right)=$ $\chi^{f}\left(a_{v f}\right)$ and $\phi(v)=\chi^{g}\left(a_{v g}\right)$ for all $v \in W(\delta)$ (recall that $\zeta^{\prime}=\zeta_{\phi}^{\prime}$ corresponds to $\left.\phi\right)$. Similarly (see (5.6) and its proof) there exists an irreducible $\mathcal{K}_{\text {-character }} \xi$ of $A_{J, w}$ such that $\eta^{\prime}\left(\tau_{w} T_{v}\right)=\xi^{f}\left(a_{v f}\right)$ and $\psi(v)=\xi^{g}\left(a_{v g}\right)$ for all $v \in W_{J}^{w} \cap W(\delta)$. 
(6.3) Proposition. With $\chi, \xi$ as above, the multiplicity of $\xi$ in the restriction of $\chi$ is equal to both the multiplicity of $\eta^{\prime} \tau_{w}$ in the restriction of $\zeta^{\prime}$ and to the multiplicity of $\psi$ $\left(\in \operatorname{Irr}_{\mu}\left(W_{J}^{w} \cap W(\delta)\right)\right)$ in the restriction of $\phi\left(\in \operatorname{Irr}_{\mu}(W(\delta))\right)$.

Proof. Let $m$ be the multiplicity of $\xi$ in the restriction of $\chi$. From the definition of $\chi$, we have that $\zeta^{\prime} \bar{f}=\chi^{f}$ and $\eta^{\prime} \tau_{w} \bar{f}=\xi^{f}$. Hence by (6.2) the multiplicity of $\eta^{\prime} \tau_{n} \bar{f}$ in the restriction of $\zeta^{\prime} \bar{f}$ equals $m$. Composing with $\bar{f}^{-1}$ (see Figure 1), this also equals the multiplicity of $\eta^{\prime} \tau_{\kappa}$ in the restriction of $\zeta^{\prime}$. Similarly, $\chi^{g}=\phi \bar{g}$ and $\xi^{g}=\psi \bar{g}$, where $\bar{g}$ is the isomorphism: $A^{g} \rightarrow \mathbf{C W}(\delta)_{\mu}$ defined by $a_{v g} \mapsto[v](v \in W(\delta))$. Thus by (6.2) $m$ is also equal to the multiplicity of $\psi$ in the restriction of $\phi$.

Application to duality theory. The concept of the dual of a character of $G$ was introduced by Curtis [4] and studied by Alvis [1]. In this section we use the explicit parametrization of the characters of $G$ in each "series" (that is, the constituents of each induced cuspidal) provided by Theorem (4.8) to describe explicitly the dual of an arbitrary irreducible character of $G$.

(7.1) Definition. The dual $\xi^{*}$ of a character $\xi$ of $G$ is defined by

$$
\xi^{*}=\sum_{J \subset I I}(-1)^{\lceil/} \operatorname{Ind}\left(P_{J} \rightarrow G ; \xi_{\left(P_{J}\right)}\right)
$$

where $\xi_{\left(P_{J}\right)}$ is the truncation of $\xi$ to $P_{J}$ (see (3.1)).

The following properties of the linear transformation * of $\operatorname{Char}(G)$ were proved by Alvis [1].

(7.2) Proposition. (i) For $J \subset \Pi$ and $\eta \in \operatorname{Char}\left(M_{J}\right)$ we have

$$
\operatorname{Ind}\left(P_{J} \rightarrow G ; \tilde{\eta}\right)^{*}=\operatorname{Ind}\left(P_{J} \rightarrow G ; \tilde{\eta}^{*}\right) .
$$

(ii) For $\xi \in \operatorname{Char}(G)$, we have $\xi^{* *}=\xi$. Thus * is an involution.

(iii) for $\xi_{1}, \xi_{2} \in \operatorname{Char}(G)$ we have $\left(\xi_{1}^{*}, \xi_{2}^{*}\right)=\left(\xi_{1}, \xi_{2}\right)$. Thus * is an isometry.

In (7.2)(i) the * occurring on the right-hand side denotes the corresponding operation on Char $\left(M_{J}\right)$. From (7.2)(iii) we see in particular that the dual of an irreducible character is an irreducible character or the negative of one.

Suppose $\xi$ is a cuspidal character of $G$. Then $\xi_{\left(P_{J}\right)}=0$ for $J \neq \Pi$, whence

$$
\text { for } \xi \text { cuspidal we have } \xi^{*}=(-1)^{|\pi|} \xi \text {. }
$$

Now consider the situation of $\S 6$ : if $\delta$ is an irreducible cuspidal character of $M_{L}$. $(L \subset \Pi)$ then combining (7.2)(i) and (7.3) gives

$$
\operatorname{Ind}\left(P_{L} \rightarrow G ; \tilde{\delta}\right) *=(-1)^{|L|} \operatorname{Ind}\left(P_{L} \rightarrow G ; \tilde{\delta}\right) .
$$

Thus the transformation * permutes (up to sign) the irreducible constituents of the "series" Ind $\left(P_{L} \rightarrow G ; \tilde{\delta}\right)$ and so to describe the action of * explicitly is to describe the associated permutation for each $L$ and $\delta$. Now Theorem (4.8) shows that these irreducible constituents are parametrized in an explicit way by $\operatorname{Irr}_{\mu}(W(\delta))$, where $W(\delta)$ is the ramification group of $\delta$ and $\mu$ is the 2-cocycle of $W(\delta)$ appearing in Theorem (3.10). Thus our description of the effect of ${ }^{*}$ on the constituents of $\operatorname{Ind}\left(P_{L} \rightarrow G ; \tilde{\delta}\right)$ will be given in terms of this parametrization. 
(7.5) THEOREM. With notation as in Theorem (4.8) let $\alpha=\alpha_{L}$ be the one-dimensional character of $W(\delta)$ defined in (5.2). Then for $\phi \in \operatorname{Irr}_{\mu}(W(\delta))$ we have

$$
\zeta_{\phi}^{*}=(-1)^{|L|} \zeta_{\alpha \phi} \text {. }
$$

The remainder of this section will be devoted to a proof of (7.5); notation will be as in the statement. The proof consists of evaluating $\left(\zeta_{\phi}^{*}, \zeta_{\phi^{\prime}}\right)$ for $\phi, \phi^{\prime} \in \operatorname{Irr}_{\mu}(W(\delta))$. By (7.1) and Frobenius reciprocity this leads to consideration of inner products of the form $\left(\operatorname{Res}\left(G \rightarrow P_{J} ; \zeta_{\phi}\right), \tilde{\eta}\right)$ where $\phi \in \operatorname{Irr}_{\mu}(W(\delta))$ and $\eta \in \operatorname{Irr}\left(M_{J}\right)$ (for various $J \subset \Pi)$. Hence we begin with

(7.6) Lemma. Let $\phi \in \operatorname{Irr}_{\mu}(W(\delta))$ and $\eta \in \operatorname{Irr}\left(M_{J}\right)$. If $\left(\operatorname{Res}\left(G \rightarrow P_{J} ; \zeta_{\phi}\right), \tilde{\eta}\right) \neq 0$ then there exists $w \in W$ with $w L \subset J$ such that $\eta$ is a constituent of

$$
\operatorname{Ind}\left(P_{w L} \cap M_{J} \rightarrow M_{J} ; \widetilde{w \delta}\right) .
$$

Proof. By the Harish-Chandra principle (3.2) applied to $M_{J}$ there is a subset $K \subset J$ and an irreducible cuspidal character $\gamma$ of $M_{K}$ such that $\eta$ is a constituent of $\theta=\operatorname{Ind}\left(P_{K} \cap M_{J} \rightarrow M_{J} ; \tilde{\gamma}\right)$. If the inner product in the statement above is nonzero then a fortiori

$$
\left(\operatorname{Res}\left(G \rightarrow P_{J} ; \zeta_{\phi}\right), \tilde{\theta}\right) \neq 0
$$

and by Frobenius reciprocity

$$
\left(\zeta_{\phi}, \operatorname{Ind}\left(P_{J} \rightarrow G ; \tilde{\theta}\right)\right) \neq 0 .
$$

But the parabolic subgroup $P_{K} \cap M_{J}$ of $M_{J}$ has unipotent radical $\left(U_{K} \cap M_{J}\right) \cdot U_{J}$. It follows that the result of lifting $\gamma$ to $P_{K} \cap M_{J}$ (from $M_{K}$ ), then inducing to $M_{J}$ and finally lifting to $P_{J}$, is the same as the result of lifting $\gamma$ to $P_{K}$ and then inducing to $P_{J}$. Thus

$$
\operatorname{Ind}\left(P_{J} \rightarrow G ; \tilde{\theta}\right)=\operatorname{Ind}\left(P_{J} \rightarrow G ; \operatorname{Ind}\left(P_{K} \rightarrow P_{J} ; \tilde{\gamma}\right)\right)=\operatorname{Ind}\left(P_{K} \rightarrow G ; \tilde{\gamma}\right) .
$$

Hence the hypothesis of the lemma implies that $\left(\zeta_{\phi}, \operatorname{Ind}\left(P_{K} \rightarrow G ; \tilde{\gamma}\right)\right) \neq 0$, whence (Ind $\left(P_{L} \rightarrow G ; \tilde{\delta}\right)$, Ind $\left.\left(P_{K} \rightarrow G ; \tilde{\gamma}\right)\right) \neq 0$. By the second part of the Harish-Chandra principle (3.3) applied to $G$, we deduce that there is an element $w \in W$ such that $M_{K}={ }^{w} M_{L}=M_{w L}$ and $w \delta=\gamma$. By (5.4) we may take $w$ to be such that $w L=K$ $(\subset J)$. The lemma follows.

(7.7) Definition. Fix $J \subset \Pi$. For each $w \in W$ such that $W L \subset J$, let $\eta(w)$ be the set of irreducible constituents of $\operatorname{Ind}\left(P_{w L} \cap M_{J} \rightarrow M_{J} ; w \delta\right)$.

By (5.5) there is a set $S_{\delta}(J)$ of representatives of the $\left(W_{J}, W(\delta)\right)$ cosets in $C_{L}(J)$ such that for each $w \in S_{\delta}(J)$ we have $w L \subset J$ and $w \Gamma^{+} \subset \Sigma^{+}$. We fix such a set $S_{\delta}(J)$ of representatives for each $J$. Let $\phi \in \operatorname{Irr}_{\mu}(W(\delta))$ be fixed also.

(7.8) LEMMA. The sets $\eta(w), w \in S_{\delta}(J)$, are pairwise disjoint, and their union includes the set

$$
\left\{\eta \in \operatorname{Irr}\left(M_{J}\right) \mid\left(\operatorname{Res}\left(G \rightarrow P_{J} ; \zeta_{\phi}\right), \tilde{\eta}\right) \neq 0\right\} .
$$

Proof. Assume $w_{1}, w_{2} \in S_{\delta}(J)$. By (3.3) applied to $M_{J}, \eta\left(w_{1}\right)=\eta\left(w_{2}\right)$ if and only if there exists $x \in W_{J}$ with ${ }^{x} M_{w_{1} L}=M_{w_{2} L}$ and $x w_{1} \phi=x w_{2} \phi$. So by (5.4) $\eta\left(w_{1}\right)=$ $\eta\left(w_{2}\right)$ if and only if $w_{2}^{-1} x w_{1} \in W(\delta)$ for some $x \in W_{J}$-that is, if and only if $w_{1}$ and 
$w_{2}$ lie in the same $\left(W_{J}, W(\delta)\right)$ double coset. Thus if $w_{1} \neq w_{2}$ then $\eta\left(w_{1}\right) \neq \eta\left(w_{2}\right)$. Moreover $\eta\left(w_{1}\right) \neq \eta\left(w_{2}\right)$ implies that $\eta\left(w_{1}\right)$ and $\eta\left(w_{2}\right)$ are disjoint (by (3.2)). The rest of (7.8) is immediate from (7.6).

We are now able to complete the proof of Theorem (7.5).

Proof of Theorem (7.5). We have

$$
\begin{aligned}
\left(\zeta_{\phi}\right)_{\left(P_{J}\right)} & =\sum_{\eta \in \operatorname{Irr}\left(M_{J}\right)}\left(\operatorname{Res}\left(G \rightarrow P_{J} ; \zeta_{\phi}\right), \tilde{\eta}\right) \tilde{\eta} \quad(\text { by }(3.1)) \\
& =\sum_{w \in S_{\delta}(J)} \sum_{\eta \in \eta\left(w^{\prime}\right)}\left(\operatorname{Res}\left(G \rightarrow P_{J} ; \zeta_{\phi}\right), \tilde{\eta}\right) \tilde{\eta} \quad(\text { by }(7.8)) \\
& =\sum_{w} \sum_{\psi}\left(\operatorname{Res}\left(G \rightarrow P_{J} ; \zeta_{\phi}\right), \tilde{\eta}_{\psi}\right) \tilde{\eta}_{\psi} \quad(\text { by }(5.8)) \\
& =\sum_{w} \sum_{\psi}\left(\operatorname{Res}\left(W(\delta) \rightarrow W_{J}^{w} \cap W(\delta) ; \phi\right), \psi\right) \tilde{\eta}_{w^{\prime} \psi} \quad(\text { by }(5.9))
\end{aligned}
$$

where in these last two lines the sums are over $w \in S_{\delta}(J)$ and $\psi \in \operatorname{Irr}_{\mu}\left(W_{J}^{w} \cap W(\delta)\right)$. We deduce that if $\phi^{\prime}$ is also an element of $\operatorname{Irr}_{\mu}(W(\delta))$ then

$$
\begin{aligned}
& \left(\operatorname{Res}\left(G \rightarrow P_{J} ; \zeta_{\phi^{\prime}}\right),\left(\zeta_{\phi}\right)_{\left(P_{J}\right)}\right) \\
& \quad=\sum_{w} \sum_{\psi}\left(\operatorname{Res}\left(W(\delta) \rightarrow W_{J}^{w} \cap W(\delta) ; \phi\right), \psi\right)\left(\operatorname{Res}\left(G \rightarrow P_{J} ; \zeta_{\phi^{\prime}}\right), \tilde{\eta}_{w^{\prime} \psi}\right) \\
& \quad=\sum_{w} \sum_{\psi}\left(\operatorname{Res}\left(W(\delta) \rightarrow W_{J}^{w} \cap W(\delta) ; \phi\right), \psi\right)\left(\operatorname{Res}\left(W(\delta) \rightarrow W_{J}^{n^{\prime}} \cap W(\delta) ; \phi^{\prime}\right), \psi\right)
\end{aligned}
$$

by a second application of Theorem (5.9). But from Lemma (2.1) we have, for any $\mu$-characters $\rho$ and $\sigma$ of $W(\delta)$,

$$
\begin{aligned}
& \left(\operatorname{Res}\left(W(\delta) \rightarrow W_{J}^{w} \cap W(\delta) ; \rho\right), \operatorname{Res}\left(W(\delta) \rightarrow W_{J}^{w} \cap W(\delta) ; \sigma\right)\right) \\
& \quad=\sum_{\psi}\left(\operatorname{Res}\left(W(\delta) \rightarrow W_{J}^{w} \cap W(\delta) ; \rho\right), \psi\right)\left(\operatorname{Res}\left(W(\delta) \rightarrow W_{J}^{w} \cap W(\delta) ; \sigma\right), \psi\right)
\end{aligned}
$$

summed over $\psi \in \operatorname{Irr}_{\mu}\left(W_{J}^{w} \cap W(\delta)\right)$. Applying this to (7.5.2) we obtain (where the sums below are over $\left.w \in S_{\delta}(J)\right)$

$$
\begin{aligned}
\left(\zeta_{\phi^{\prime}}, \operatorname{Ind}\left(P_{J}\right.\right. & \left.\left.\rightarrow G ;\left(\zeta_{\phi}\right)_{\left(P_{J}\right)}\right)\right)=\left(\operatorname{Res}\left(G \rightarrow P_{J} ; \zeta_{\phi^{\prime}}\right),\left(\zeta_{\phi}\right)_{\left(P_{J}\right)}\right) \\
& =\sum_{w}\left(\operatorname{Res}\left(W(\delta) \rightarrow W_{J}^{w} \cap W(\delta) ; \phi\right), \operatorname{Res}\left(W(\delta) \rightarrow W_{J}^{w} \cap W(\delta) ; \phi^{\prime}\right)\right) \\
& =\sum_{w}\left(\phi^{\prime}, \operatorname{Ind}\left(W_{J}^{w} \cap W(\delta) \rightarrow W(\delta) ; \operatorname{Res}\left(W(\delta) \rightarrow W_{J}^{w} \cap W(\delta) ; \phi\right)\right)\right) \\
& =\sum_{w}\left(\phi^{\prime}, \phi \operatorname{Ind}\left(W_{J}^{w} \cap W(\delta) \rightarrow W(\delta) ; 1\right)\right) \quad(\text { by }(2.1)(\mathrm{iv})) .
\end{aligned}
$$


Hence (7.1) gives

$$
\begin{aligned}
\left(\zeta_{\phi^{\prime}}, \zeta_{\phi}^{*}\right) & =\left(\phi^{\prime}, \phi \sum_{J} \sum_{w}(-1)^{|/|} \operatorname{Ind}\left(W_{J}^{w} \cap W(\delta) \rightarrow W(\delta) ; 1\right)\right) \\
& \left.\quad \text { (sums over } J \subset \Pi \text { and } w \in S_{\delta}(J)\right) \\
& =\left(\phi^{\prime},(-1)^{|L|} \alpha \phi\right) \quad(\text { by }(5.3)) .
\end{aligned}
$$

This proves Theorem (7.5).

\section{REFERENCES}

1. Dean Alvis, The duality operation in the character ring of a finite Chevalley group, Bull. Amer. Math Soc. 1 (1979), 907-911.

2. N. Bourbaki, Algèbre commutatif, Hermann, Paris, 1964, Chapters 5, 6.

3. C. W. Curtis, Truncation and duality in the character ring of a finite group of Lie type, J. Algebra 62 (1980), 320-332.

4. Reduction theorems for characters of finite groups of Lie type, J. Math. Soc. Japan 27 (1975), $666-688$.

5. C. W. Curtis, N. Iwahori and R. Kilmoyer, Hecke algebras and characters of parabolic type of finite groups with BN-pairs, Inst. Hautes Etudes Sci.Publ. Math. 40 (1971), 81-116.

6. C. W. Curtis and I. Reiner, Representation theory of finite groups and associative algebras, Interscience, Wiley, New York, 1962.

7. R. B. Howlett and G. I. Lehrer, A comparison theorem and other formulae in the character ring of $a$ finite group of Lie type , Papers in Algebra, Analysis and Statistics (Proc. 21st Austral. Math. Soc. Summer Res. Inst.), Contemporary Math., vol. 9, Amer. Math. Soc., Providence, R.I., 1982, pp. 285-289.

8. Duality in the normalizer of a parabolic subgroup, Bull. London Math. Soc. 14 (1982), $133-136$.

9. , Induced cuspidal representations and generalized Hecke rings, Invent. Math. 58 (1980), $37-64$

10. G. Lusztig, Irreducible representations of finite classical groups, Invent. Math. 38 (1976), 101-159.

11. N. Iwahori, Generalized Tits systems (Bruhat decomposition) on p-adic semisimple groups, Proc. Sympos. Pure Math., vol. 9, Amer. Math. Soc., Providence, R.I., 1966, pp. 71-83.

12. K. McGovern, Multiplicities of principal series representations of finite groups with split $(B, N)$ pairs, J. Algebra 77 (1982), 419-442.

Department of Pure Mathematics, Institute of Advanced Studies, Australian National University, Canberra, A.C.T. 2600, Australia

Department of Pure Mathematics, University of Sydney, New South Wales 2006, Australia 